\title{
Quantum mechanics as a deterministic theory of a continuum of worlds
}

\author{
Kim Joris Boström(D)
}

Received: 30 October 2014 / Accepted: 3 June 2015 / Published online: 23 June 2015

(C) Chapman University 2015

\begin{abstract}
A non-relativistic quantum mechanical theory is proposed that describes the universe as a continuum of worlds whose mutual interference gives rise to quantum phenomena. A logical framework is introduced to properly deal with propositions about objects in a multiplicity of worlds. In this logical framework, the continuum of worlds is treated in analogy to the continuum of time points; both "time" and "world" are considered as mutually independent modes of existence. The theory combines elements of Bohmian mechanics and of Everett's manyworlds interpretation; it has a clear ontology and a set of precisely defined postulates from where the predictions of standard quantum mechanics can be derived. Probability as given by the Born rule emerges as a consequence of insufficient knowledge of observers about which world it is that they live in. The theory describes a continuum of worlds rather than a single world or a discrete set of worlds, so it is similar in spirit to many-worlds interpretations based on Everett's approach, without being actually reducible to these. In particular, there is no splitting of worlds, which is a typical feature of Everett-type theories. Altogether, the theory explains (1) the subjective occurrence of probabilities, (2) their quantitative value as given by the Born rule, and (3) the apparently random "collapse of the wavefunction" caused by the measurement, while still being an objectively deterministic theory.
\end{abstract}

Keywords Foundations of quantum mechanics - Intepretation of quantum mechanics - Bohmian mechanics . Many-worlds theory $\cdot$ Continuous substance $\cdot$ Mode of existence

\section{Contents}

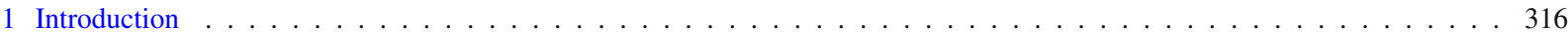

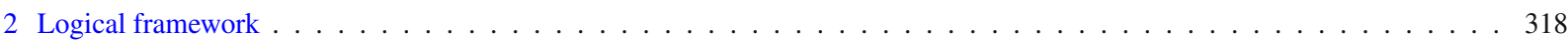

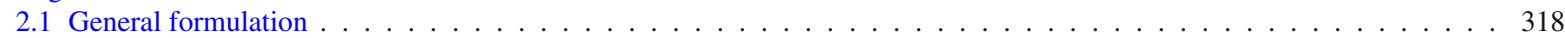

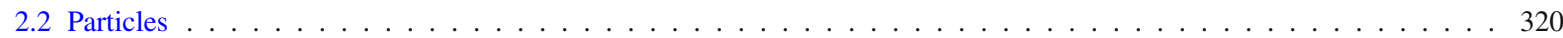

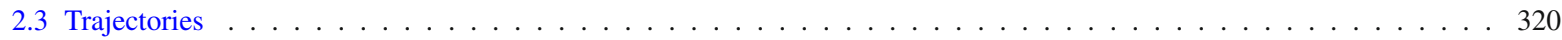

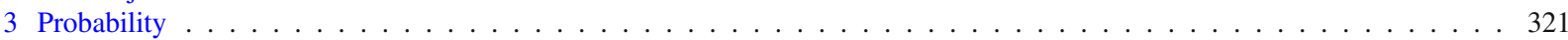

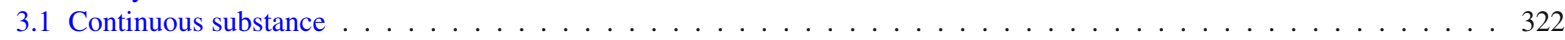

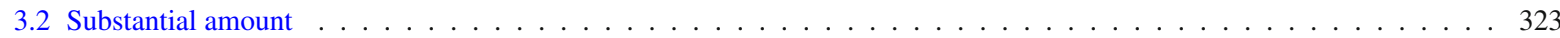

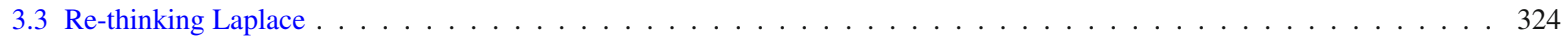

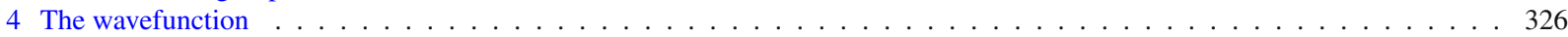

K. J. Boström $(\varangle)$

University of Münster, Münster, NRW, Germany

e-mail: mail@kim-bostroem.de 


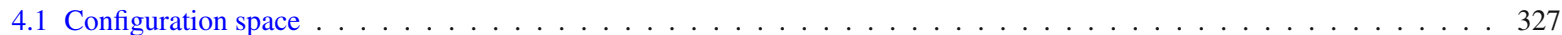

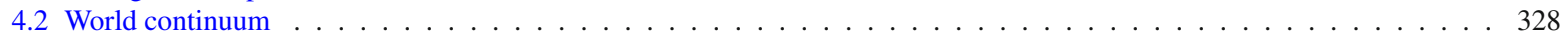

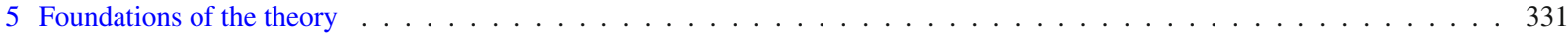

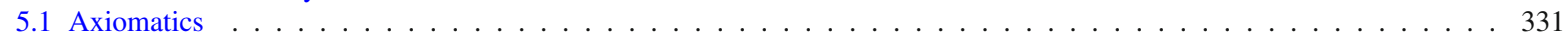

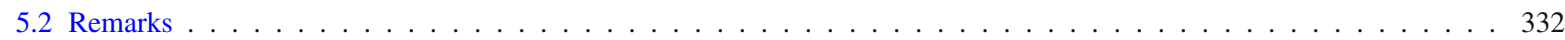

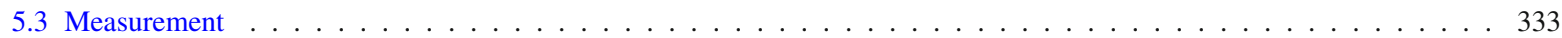

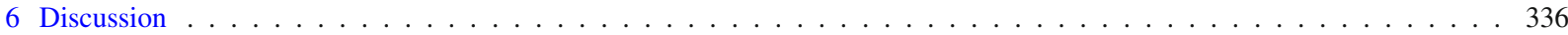

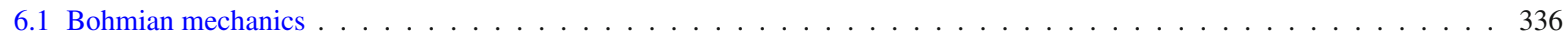

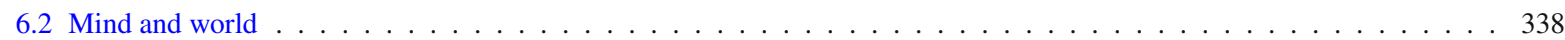

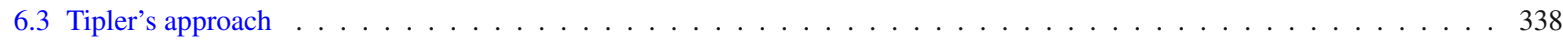

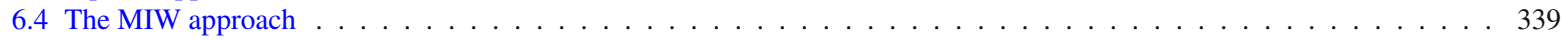

6.5 Newtonian $\mathrm{QM} \ldots \ldots \ldots \ldots \ldots \ldots$

6.6 Poirier and Schiff's approach . . . . . . . . . . . . . . . . . . . . . . . . . . . . . 342

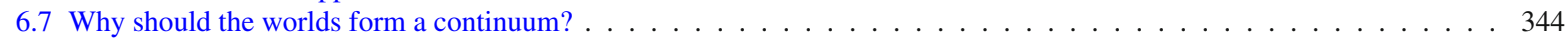

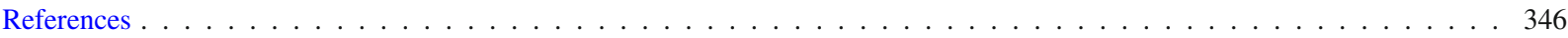

\section{Introduction}

The ideas proposed in this paper have grown out of dissatisfaction with the existing interpretations of quantum mechanics. The problem is not that quantum mechanics does not yield the correct experimental predictions, but rather that there is still no consensus about the metaphysical content of the theory, that is, the story that quantum mechanics tells us about reality. Some people simply turn the tables and consider the lack of a clear, indisputable metaphysical interpretation not as a bug but rather a feature, denying the existence of objective reality altogether. Doing so, however, appears to me as an act of resignation rather than a satisfying solution to the conundrum.

The theory that I am going to propose offers a transparent and consistent interpretation of non-relativistic quantum mechanics. Measurements are taken to be ordinary processes, there is no objective "collapse of the wavefunction", and the wavefunction is a convenient mathematical representation of a physically existing continuum of trajectories through configuration space, each one corresponding to an individual world. There is no distinction between a "quantum system" and a "classical apparatus" to explain the definite outcome of measurements and their probabilistic behavior. Elementary particles have at all times and in all worlds a well-defined position in 3D space; there are no such things as "probability clouds" and "objective uncertainty". Rather, probability emerges as the consequence of insufficient knowledge of observers about which world it is that they live in. The quantitative form of such epistemic probability does not rely on a "quantum equilibrium hypothesis" as in Bohmian mechanics, or a "branch weight" as in Everettian mechanics, but is derived from the concept of a substantial density of trajectories in configuration space, which is regarded as an objective feature of the physically existing universe (or multiverse, if one prefers to say). More precisely, the universe (multiverse) is conceived of as a continuum of trajectories endowed with a certain density that determines how densely the trajectories are packed in different regions of configuration space. Each trajectory corresponds to a world, and all worlds equally exist. My proposal is different from Bohmian mechanics in that the wavefunction does not represent a physical field existing in addition to particles, and it is different from Everettian mechanics in that the worlds are precisely defined and do not split (Fig. 1). The theory is based on ideas initially published as a preprint draft [5], which has been completely re-worked and enhanced, in particular by adding a logical framework to properly deal with propositions about physical systems in a multiplicity of worlds, and by providing the conceptual prerequisites for treating the collection of worlds as a continuous substance. After having finished and submitted an earlier version of this manuscript, I noticed that essentially the same theory, though with a stronger focus on formal aspects and less focus on ontological and epistemological matters, has independently been put forward by Poirier and Schiff [35,39]. Although already having been aware of, and having cited, these publications, I did not fully recognize how close their theory was to mine. The similarities have probably been masked from my eyes by the authors' central emphasis on the elimination of the wavefunction from the theory, which was (and is) not an issue in my approach. The wavefunction in my approach is a generating function of, and thus a mathematical representative for, a continuum of trajectories identified as worlds, and so it has its own 


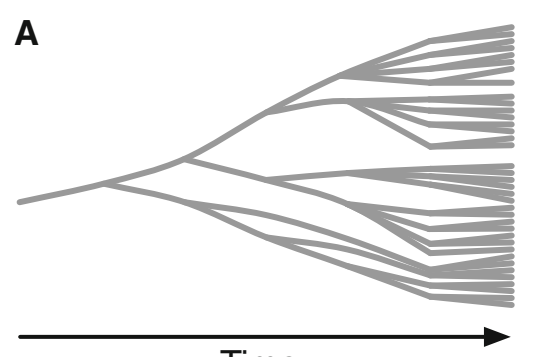

Time

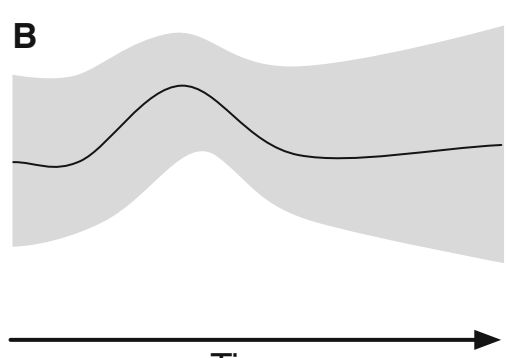

Time

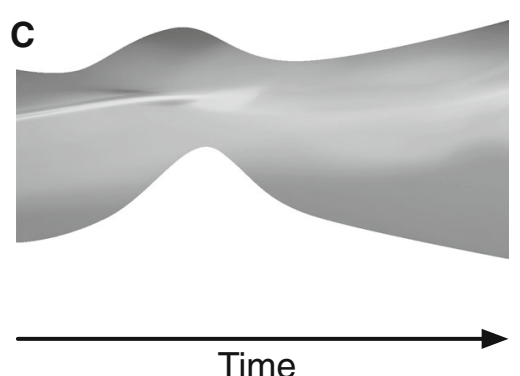

Time

Fig. 1 Schematic illustration of the different ontologies of Everettian mechanics (a), Bohmian mechanics (b), and the here-proposed theory (c). Everettian mechanics describes a universe of constantly branching, vaguely defined worlds that evolve through Hilbert space. Bohmian mechanics describes a wavefunction plus one single precisely-defined world that both evolve through configuration space. The here-proposed theory describes the a continuum of precisely defined worlds of varying density, which evolves through configuration space, and where each world corresponds to a Bohmian trajectory. The wavefunction serves as a generating function for the continuum of trajectories, which constitutes the physically existing universe

justification to remain within the theory. I will discuss the approach by Poirier and Schiff in some more detail in the last section, along with related approaches by [21,40,43].

The remaining part of the paper is structured as follows. In Sect. 2, a logical framework is proposed to properly deal with propositions evaluated in a multiplicity of worlds and at a multiplicity of times, whereby time and world are treated as so-called modes of existence. The concept of a world continuum is introduced as a physically existing entity consisting of a continuum of worlds. Also, the concept of an instance is introduced, which comes in three flavors. A time-instance is an instance of an object at a specific time, a world-instance is an instance of an object in a specific world, and a time-world-instance is an instance of an object at both a specific time and in a specific world. The logical framework is then applied to the case of particles as the basic entities of the theory. It is postulated that each world corresponds to one and only one trajectory of the universe in configuration space, so that worlds can be identified with trajectories. Each time-world-instance of the universe corresponds to the configuration of all particles in the universe at a specific time and in a specific world.

In Sect. 3, the notion of continuous substance is introduced and discussed to properly deal with a continuum of worlds later on. In particular, the substantial amount and the substantial density of a given substance are defined and discussed. Subsequently, probability is put forward as an epistemic concept deriving from the insufficient knowledge of observers about which world it is that they live in. To this aim, the well-known Laplacian rule is generalized to a continuous number of possibilities with the help of a measure that is specified as the substantial amount of worlds whose trajectories are crossing a certain region in configuration space.

The role and the physical meaning of the wavefunction is discussed in Sect. 4, as well as the role and the meaning of configuration space and of the world continuum. It is held that the wavefunction does not represent a physically existing entity itself but is rather to be considered an abstract generating function for the physically existing world continuum.

A minimal set of postulates is given in Sect. 5 to formally define the theory. Measurement is introduced as a special case of an otherwise ordinary interaction between a system of interest and a measurement apparatus, yielding the Born rule as a subjective measure of uncertainty about measurement outcomes obtained in individual worlds. The "collapse of the wavefunction" is derived as a useful but merely subjective description at the level of an individual world.

In the final section, several aspects of my proposal and their relation to other proposals and criticisms found in the literature are discussed. In particular, the relation between objective reality and subjective experience in the presence of a multiplicity of worlds is addressed. My proposal is compared to Bohmian mechanics, to Tipler's formulation of quantum mechanics [43], to the MIW approach of Hall et al. [21], to Sebens' Newtonian QM [40], and to Poirier and Schiff's approach [35,39]. I will also respond to criticisms raised by [40,48] against the idea of a continuum of worlds. 


\section{Logical framework}

After having long been ignored and ridiculed, the many-worlds interpretation has in recent years become a scientifically recognized and intensely discussed interpretation of quantum mechanics (see $[42,46,54]$, for modern accounts). Among the main objections against the many-worlds interpretation is the criticism that it is too vague about the notion of worlds, and that the entire conception of many worlds existing in parallel is absurd in the first place (cf. [2,24]). Indeed, at first sight it appears problematic or even absurd to consider our world as one out of many worlds, for we usually speak of the world and understand it as everything that exists. The inventor of many-worlds quantum theory, Hugh Everett III, originally did not use the term "world", and he named his theory the "relative-state formulation of quantum mechanics" [17] and "the theory of the universal wavefunction" [18]. However, the notion of worlds became more and more popular in discussions of Everett's theory (cf. [2,56]) and was eventually introduced to the public by DeWitt [12]. I will stick to the now firmly established convention of using the term "world", though I will use it in a very specific manner. In the extended logical framework that I will propose, there are two modes of existence, and these are time and world. The key idea is very simple. In conventional logic, objects cannot at the same time have a property and not have that property. In the proposed logical framework, objects cannot at the same time and in the same world have a property and not have that property. So, while in conventional logic there is only one mode of existence, which is time, in the extended framework there are two modes of existence, which are time and world. We will first concentrate on the time mode and suppress the world mode. Once we have clarified how to treat time in a logical framework, and once we have set up the terminology, we can straightforwardly extend the framework to also include the world mode. We shall see that world and time are treated in an analogous manner, and since we are already quite familiar with the concept of time - at least we have some intuition in using this concept - we will be able to understand how the concept of world can be understood as well. In a similar sense that classical mechanics can be understood as a many-times theory, non-relativistic quantum mechanics can be understood as a many-worlds theory in addition to its many-times aspect.

Why should one take it that quantum mechanics, in contrast to classical mechanics, needs a world mode of existence in addition to the time mode? Because in quantum mechanics the worlds interfere with each other. This interference of worlds is responsible for the typical "quantum" phenomena that go beyond classical explanation. For example, an electron going through a double-slit produces an interference pattern because its trajectory in one world interferes with its trajectories in other worlds. In the classical theory there are no interference patterns produced by individual particles, so there is no need to consider additional worlds that interfere with each other. Other experimental paradigms where a many-worlds interpretation yields a straightforward and transparent explanation is neutron interference [45], quantum computation [11], and counterfactual measurement [16,44,47]. After all, the most important reason to favor a many-worlds interpretation is not simply to satisfy a somewhat romantic attitude but rather to better understand quantum phenomena and to avoid serious conceptual difficulties.

\subsection{General formulation}

Let us begin with the more familiar concept of time. Physically existing objects have their properties each at a given instance of time. An instance of time is also referred to as a time point, but in the following we shall drop the explicit distinction between time points and times whenever there is no risk of confusion. That is, by saying that an object $o$ has a particular property $F$ at a given time $t$, we mean that the object has the property at the time point $t$. We denote this statement by the formula $p(t)=$ " $F o @ t$ " and call it an anchored proposition. As usual in a physical theory, time points are represented by real numbers, so the set $\mathcal{T}$ of all time points equals the entire real line, $\mathcal{T}=\mathbb{R}$. The temporal anchor plays the role of the present time of an anchored proposition. Consider an unanchored proposition like "the traffic lights are green". In one moment the traffic lights may be green, in another moment they may be red, or yellow, or something else. We can anchor an unanchored proposition by adding "now" to it, where "now" is an unspecified temporal anchor to be treated as a variable. Formally, the partial expression " $F o$ " occurring in the proposition $p(t)=$ "Fo @ $t$ " is the body of the proposition, and $t$ is the (temporal) anchor. If the temporal anchor is 
unspecified, that is, it is not given by a real number but remains a variable, then the proposition remains unevaluated. We can evaluate it either by specifying a numerical value for $t$, or by quantifying over $t$, so that, for example, for a given time period $T \subset \mathcal{T}$ the proposition " $\forall(t \in T): F o @ t$ " is understood as "object $o$ has property $F$ during $T$ ", which is either true or false. The proposition is then anchored to the specified interval $T$, which may also coincide with the entire time domain $\mathcal{T}$. In the latter case we may use the shorthand notation " $\forall t$ " to mean " $\forall(t \in \mathcal{T})$ ", and similarly " $\exists t$ " to mean " $\exists(t \in \mathcal{T})$ ".

Since real numbers form an ordered set, a temporal anchor $t$ can be set into relation with other time points, so that time points that are smaller or bigger than $t$, in the usual order of $\mathbb{R}$, play the role of, respectively past (earlier) or future (later) time points relative to $t$. We then can then formalize the proposition "Object $o$ will have the property $F$ " as " $\exists\left(t^{\prime}>t\right): F o @ t^{\prime}$ ", and the proposition "Object $o$ never had the property $F$ " as " $\forall\left(t^{\prime}<t\right): \sim F o @ t^{\prime}$ ". The variable $t$ is then the unspecified anchor of both propositions and represents the "now" of the proposition, that is, the present time relative to which the body of each proposition is anchored in the future and the past, respectively.

Let us now extend the logical framework by adding world as another mode of existence. That is, an object has properties only with respect to a particular time and a particular world. Anchored propositions are then of the form $p(t, w)=$ " $F o @(t, w)$ ", which is understood as "object $o$ has property $F$ at time $t$ and in world $w$ ". The expression " $F o$ " is, again, the body of the anchored proposition $p(t, w)$, and the expression "@ $(t, w)$ " is the anchor, with $t$ being the temporal anchor and $w$ being the world anchor. Just like the temporal anchor $t$ plays the role of the present time of the proposition, the world anchor $w$ plays the role of the actual world of the proposition. Let us denote the set of all worlds by $\mathcal{W}$ and allow to quantify over both worlds and times, so that, say, for a given set $W \subset \mathcal{W}$ the formal proposition $p(t)=" \forall(w \in W) \exists\left(t^{\prime}<t\right): F o @\left(t^{\prime}, w\right)$ " reads "for all worlds in $W$ the object $o$ once had the property $F$ ". That proposition involves the variable $t$ as an unspecified temporal anchor, and it remains unevaluated until $t$ is specified or quantified. Consider another different example involving the variable $w$ as an unspecified world anchor: the formal proposition $p(w)=" \forall t: F o @(t, w)$ " reads "in the actual world the object $o$ always has the property $F$ " and remains unevaluated as long as the actual world $w$ is not specified or quantified. If the actual world of the proposition lies within some given set $W \subset \mathcal{W}$, then the proposition becomes $p=" \forall t \forall(w \in W): F o @(t, w)$ ", which is understood as "in all worlds contained within $W$ the object $o$ always has the property $F$ ". The proposition $p$ contains no free variables and hence is an evaluated proposition. In the case $W=\mathcal{W}$ we may shorten " $\forall(w \in \mathcal{W})$ " into " $\forall w$ ", and " $\exists(w \in \mathcal{W})$ " into " $\exists w$ ".

There is yet another, logically equivalent, way of understanding anchored propositions, which might be favorable under certain circumstances. The occurrence of an object at a particular time and in a particular world can be considered an instance of that object. Then, there are different instances of one and the same object at different times and in different worlds, but it is still the same object that is instantiated. In terms of instances, thus, the formula $p(t, w)=$ “Fo@( $t, w)$ " is understood as "the instance $o @(t, w)$ has the property $F$ ”. Note that the talk of instances and the talk of objects are just two different ways to read a proposition. We may switch back and forth between the two readings as we like and keep in mind that doing so does not affect the content of the proposition. There are three different ways of using instance talk. One of these ways is the talk of time-world instances that we have just encountered. Another way is to translate only the time mode into an instance and leave the world mode as it is. Thus, instead of speaking of an object having some property at a specific time and in a specific world, one may speak of a time-instance of the object having the property in a specific world. So, for example, instead of saying that "Joe went to school when he was six in world $w$ ", one may say that "six-year-old Joe went to school in world $w$ ". Formally, a time-instance of Joe is denoted as Joe $t \equiv$ Joe@ $t$, and in the given example $t$ would denote some point in time when Joe was 6 years old. The third way is to translate only the world mode into an instance, so that $\mathrm{Joe}_{w} \equiv$ Joe @ $w$ is the instance of Joe in world $w$.

It is helpful to visualize objects as being extended into both the time mode and the world mode. That is, one may conceive of modes in a manner analogous to dimensions. Instances then collapse objects along some of their modes. A time-instance collapses the object along the time mode, so that it remains extended along the world mode. For example, a time-instance of an electron is an electron cloud. A world-instance collapses the object along the world mode, so that it remains extended along the time mode. For example, a world-instance of an electron is a trajectory. Last, a time-world-instance collapses the object along both modes, so that it loses its modal extension and becomes 
a "modal point". For example, a time-world-instance of an electron is a point located in 3D space. Instances inherit the properties that the objects have at their instantiation. For example, a time-world instance $e_{t, w}$ of an electron $e$ is located at the position $\boldsymbol{x}$ that the electron occupies at time $t$ in world $w$. And as for Joe, his time-world instance $\mathrm{Joe}_{t, w}$ has the property of going to school.

It should have become clear from the setup of the framework and the chosen formulations that I understand neither the present time nor the actual world as absolute entities. Rather, I regard all times and all worlds as equally existing, although they do not exist in the same sense in which objects exist. An object exists at a time and in a world. Without a time and a world the object cannot exist. So times and worlds are preconditions of existence, or more precisely, in the terminology of the here-proposed framework, times and worlds are modes of existence. ${ }^{1}$

\subsection{Particles}

As usual in a physical theory, we will from now on restrict the set of objects to systems of elementary particles. Technically, a system of particles is a mereological sum of particles, that is, particles are in a part-whole relationship with systems. A one-particle system consists of only one particle, and an $N$-particle system consists of $N$ particles. If a particle is part of a system which is part of another system, then the particle is also part of the latter system. We shall not dive into the details of the theory of mereology, and the reader may be referred to textbook literature (cf. [52]).

Systems of particles cannot be naively identified with macroscopic objects. Macroscopic objects (ships, dogs, people), may lose or gain particles and still remain the same macroscopic object, which is not the case for systems. It is a highly nontrivial question how to define macroscopic objects and how to identify them across time. Of course, it should be no less problematic to identify macroscopic objects across worlds. If in one world Joe is lacking one hair compared to our world, it would appear natural to assume that it is still Joe who is lacking a hair in that world, and not a completely different person. I believe these issues can somehow be settled, so that it makes sense to speak of one and the same object (including people) across different times and across different worlds. Luckily, though, these difficulties do not affect the quantum theory that I am going to propose, as they do not arise for elementary particles. An elementary particle at different times simply is the same particle, only at different times, and a particle in different worlds simply is the same particle, only in different worlds. (Recall that instances are no copies; it is the same particle that only occupies different positions in space at different times and in different worlds.)

The symbol " $o$ " denoting a system of particles in a proposition like " $F o @(t, w)$ " is taken as a rigid designator in a sense analogous to how Kripke [25] introduced the term into modal logic: the symbol refers to the same system at all times when it exists, and in all worlds where it exists. Since we stay in the non-relativistic domain, there is no particle creation or annihilation. Thus, if a particle exists in one world at one time, then it exists at all other times in that world. Let us further demand particle conservation across worlds, so that when a system $o$ exists at one time and in one world, it also exists at any other time and in any other world. There can be no particles missing and no particles being added to the system across worlds and across time. The system of all particles is identified with the universe, and the number of particles in the universe is assumed to be finite and equal to some fixed number $N$.

\subsection{Trajectories}

Particles have a fundamental property, which is their position in three-dimensional space. A particle can at some time $t$ and in some world $w$ have the position $\boldsymbol{q}$ and at another time $t^{\prime}$ and in another world $w^{\prime}$ have the position

\footnotetext{
${ }^{1}$ It should be remarked that the framework is, with regard to the theory of relativity, a non-relativistic framework, because it treats time not on the same footing as space. In a relativistic framework to be developed in a future publication, time as an anchor of propositions must be replaced by a spacelike hypersurface in four-dimensional spacetime.
} 
$\boldsymbol{q}^{\prime}$. Let $u$ be the universe consisting of $N$ particles, and $q=\left(\boldsymbol{q}_{1}, \ldots, \boldsymbol{q}_{N}\right)$ be a complete list of the positions of all particles. Then this list of positions, which is called a configuration of the universe, can mathematically be interpreted as a vector in the $3 N$-dimensional space $\mathcal{Q}:=\mathbb{R}^{3 N}$, which is called the configuration space. A point in the $3 N$-dimensional configuration space corresponds to the position of $N$ particles in the three-dimensional space ( $3 D$ space, in short). There is a subtle but profound issue with defining the configuration space as the vector space $\mathbb{R}^{3 N}$, and this issue will later be discussed and addressed by re-defining the configuration space as the tensor space $\mathbb{R}^{3 \times N}$ instead. The following considerations, though, are independent of this choice of definition.

Let $F_{q}$ be the property of having the configuration $q \in \mathcal{Q}$; then the proposition " $F_{q} u @(t, w)$ " asserts that the universe has the configuration $q$ at time $t$ and in world $w$. Now let us assert that at every time there is for every world a unique configuration of the universe. In propositional calculus this is translated into the conjunction of the following two propositions:

$$
\begin{aligned}
\forall(t, w) \exists q: & F_{q} u @(t, w) \\
\forall\left(t, w, w^{\prime}, q, q^{\prime}\right): & \left(F_{q} u @(t, w) \wedge F_{q^{\prime}} u @\left(t, w^{\prime}\right)\right) \rightarrow\left(q=q^{\prime} \leftrightarrow w=w^{\prime}\right) .
\end{aligned}
$$

The first proposition asserts that for every time and for every world there is (at least) one configuration of the universe, and the second proposition asserts that the configuration is unique. So there is a set of trajectories $\Gamma=\{\gamma\}$ through configuration space, which is in one-to-one correspondence to the set of worlds $\mathcal{W}=\{w\}$,

$w \leftrightarrow \gamma$.

Consequently, we may identify worlds and trajectories. This should not be taken to suggest that worlds literally are trajectories, in the sense of a synonym. The term "world" just has a different linguistic function than the term "trajectory". But whenever the term "world" is used in the theory, we know that it corresponds to a unique trajectory. With regard to the considerations further above, a trajectory is a world-instance of the universe, that is, it is fixed to one specific world but extended into the time mode. A time-world-instance of the universe would then be a particular configuration of the universe.

The uniqueness relations (1) and (2) imply that each trajectory is uniquely defined by any one of its points. So, we may parametrize the trajectories by their initial configuration at $t=0$, yielding the trajectory function $\xi=\xi_{t}(q)$, so that each trajectory $\gamma$ is related to the trajectory function via $\gamma_{t}=\xi_{t}\left(\gamma_{0}\right)$, which implies that $\xi_{0}(q)=q$. The trajectory function is a unique characterization of the continuous bundle of trajectories $\Gamma$ and hence can be regarded as an alternative representation of the latter.

Up to now there have been no further restrictions imposed on the actual form of the trajectories. They are not required (so far) to be continuous or differentiable or anything else; they might be erratic and discontinuous, jumping around across configuration space from one moment to another in a fractal manner, resembling a random process rather than a deterministic evolution. The actual form of the trajectories is what the physical laws will yield. These laws are sorting out unphysical trajectories from physical ones, which leads us to a quite general definition of a physical law: a physical law is a restriction on the set of logically possible ways the universe may evolve in time. There are (vastly) more logically possible ways the universe may evolve than there are physically possible ways. Consequently, the set of logically possible worlds is much bigger than the set of physically possible worlds. A physical theory essentially defines how the set of logically possible worlds is to be restricted to the set of physically possible worlds by conditions imposed on the corresponding trajectories, and these conditions come in the form of differential equations.

\section{Probability}

The success of quantum mechanics is often regarded as evidence for objective probability and objective uncertainty. Quantum mechanics, so we are told, enforces a picture of Nature not being decided about "how to proceed" when a measurement takes place, or even not being decided about "how to be" with respect to unobserved system properties. However, Bohmian mechanics as well as Everettian mechanics offers a different picture in this respect. 
Both theories deny the necessity of objective probability. Rather, the universe is at all times in a certain (quantum) state, and probability only comes into play as a consequence of incomplete knowledge of the observer, a concept of probability that is referred to as subjective or epistemic probability. In Everettian mechanics, the world of an observer is splitting up into multiple worlds during a measurement process, and the observer does not know which world he or she (as a macroscopic system capable of conscious experience) will end up in after the measurement. There are puzzling issues with this kind of interpretation (cf. [24,30,38,41,55]), but we will not get into detail here. In Bohmian mechanics, the particles in the universe are at all times in a certain configuration, but the observer does not (precisely) know which configuration that is. The challenge of both interpretations is not merely a philosophical one, but also how to get the empirically correct numerical values for the probabilities as given by Born's rule. For $\hat{\Pi}_{a}$ being a projector onto the eigenspace of some eigenvalue $a$ of an observable $\hat{A}$, Born's rule says that for a system being at time $t$ in a state described by the wavefunction $\Psi_{t}$, the probability to find the observable $\hat{A}$ obtaining the value $a$ in a measurement at time $t$, is given by

$P_{t}(a)=\frac{\left\|\hat{\Pi}_{a} \Psi_{t}\right\|^{2}}{\left\|\Psi_{t}\right\|^{2}}$.

As for Everettian mechanics, there are numerous attempts to derive Born's rule using various approaches involving observer memory states [17,18], infinite ensembles [22], frequency operators [19], decoherence [37,55], consistent histories [13,33], and decision theory [10,36]. All these approaches are still controversial. As for Bohmian mechanics, Born's rule is derived on the basis of an additional quantum equilibrium hypothesis, whose status and justification are a controversial issue as well. The quantum equilibrium hypothesis asserts that the probability density of the configuration of a system described by the wavefunction $\Psi_{t}$ is at some time point $t$ given by

$\rho_{t}(q)=\frac{\left|\Psi_{t}(q)\right|^{2}}{\int d q^{\prime}\left|\Psi_{t}\left(q^{\prime}\right)\right|^{2}}$

in which case the system is said to be in quantum equilibrium. By virtue of the continuity equation, then, the probability distribution $\rho_{t}$ is guaranteed to satisfy relation (5) for all times $t$, a feature that is called equivariance. As the here-proposed theory, which may be called the world continuum theory, is a combination of Bohmian and Everettian mechanics, it may come to no surprise that it avoids objective probability in favor of an epistemic account. Probability comes into play as a measure of the subjective uncertainty of an observer about the world he or she actually lives in, which is a somewhat sloppy way of saying that he or she does not precisely know which world mode is to be used when determining the trajectory of the universe that governs his or her own experience. We will use that sloppy talk since it is much shorter and simpler to grasp, but we shall keep in mind that it has to be understood in a more sophisticated sense.

\subsection{Continuous substance}

The approach to probability that I am going to propose is based on the concept of continuous substance, which might appear straightforward or even trivial to some readers, yet unfamiliar or even absurd to others. The idea of continuous substance has somewhat come out of fashion. Many of us have become so highly accustomed to the concept of discrete particles, quantum jumps, and energy quanta, that they can hardly imagine continuous substances. To them, the very concept of measuring the quantity of existing stuff is rigidly tied to its discrete nature. There is, so they believe, more stuff of a particular kind contained within some region $X$ than there is stuff contained within another region $X^{\prime}$ if and only if there are more particles of that stuff in $X$ than there are in $X^{\prime}$. In contrast, children do in general not have any problems when comparing the quantity of different heaps of continuous stuff. "Bob has more ice cream than me", little Alice may be heard to complain, and we can hardly expect her to be aware of the discrete structure of matter. In her mind there seems to be an intuitive concept of a "more" relation between heaps of stuff that is not rigidly tied to the counting of particles. To this intuitive concept I would like to appeal. 


\subsection{Substantial amount}

In the case of a discrete substance we can compare two heaps of substance by counting and comparing the number of discrete constituents (particles) of the heaps. This is not possible in the case of continuous substance. Anyway, the notion of comparing quantities of substance should be applicable also in this case. If there is continuous substance distributed in space, then there should be a sense in which there is more substance contained within some region in space than there is contained within another region. Being able to compare quantities of substance is a minimal requirement to justify the notion of substance itself. Note that by substance we do not necessarily mean matter. Substance, in the sense that we shall be using the term here, simply means anything that is objectively existing in physical space. It might be matter, but it might as well be energy or an electromagnetic field. If there were such thing as an objective probability distribution in space, then it would constitute a substance, too.

Let us leave open the question whether the physical space is the traditional $\mathbb{R}^{3}$ or rather the configuration space or still some other space. For the time being, all we require is that the physical space $\mathcal{X}$ is a Lebesgue-measurable vector space. The Borel sets of $\mathcal{X}$ form a $\sigma$-algebra and they are called measurable subsets of $\mathcal{X}$. When we speak of subsets of $\mathcal{X}$ in the following, we shall mean measurable subsets.

Let there be a continuous substance $S$ distributed in space by a density function $\rho \geq 0$ over $\mathcal{X}$. We may think of $S$ as a continuous fluid or gas. At each point $x$ in space where $\rho(x)>0$ let us hold that there is $S$, and at those points where $\rho(x)=0$ let us hold that there is no $S$. Moreover, if for two points $x$ and $x^{\prime}$ we have $\rho(x)=c \cdot \rho\left(x^{\prime}\right)$, then we hold that $S$ is $c$ times more densely packed within an infinitesimal volume centered at $x$ than it is packed at $x^{\prime}$. Put simply, $\rho$ measures not the density of a physical quantity like mass, charge, or energy, which is associated with a physical unit, but rather the density of the substance itself; let us, therefore, call it the substantial density. With $\mathrm{d} x$ denoting the infinitesimal volume element in $\mathcal{X}$, the integration of $\rho$ over some finite region $X$ in space,

$\mu(X)=\int_{X} \mathrm{~d} x \rho(x)$,

yields the substantial amount of $S$ contained within that region. So, instead of asking how many discrete constituents of stuff are there, as we may do in the case of discrete substances, we more generally ask how much of the stuff is there, which is then answered by the substantial amount. While $\mu$ is dimensionless, the density $\rho$ has the dimension of substance per unit volume and its SI units are $1 / m^{D}$, where $D$ is the dimension of the physical space. We may calibrate the measure $\mu$ as we like. For example, we may calibrate $\mu$ to a reference amount $\mu_{0}=\mu\left(X_{0}\right)$ for some region $X_{0}$, such that the calibrated measure $\tilde{\mu}=\mu / \mu_{0}$ would measure the amount of substance in multiples of the reference amount $\mu_{0}$ contained within the reference region $X_{0}$. If the total amount of substance is finite, we may most conveniently calibrate the measure $\mu$ to $\tilde{\mu}=\mu / \mu(\mathcal{X})$, which means that $\tilde{\mu}$ would measure the overall proportion of substance contained within a given region.

The substantial density is not actually more fundamental than the substantial amount. We could as well have started from the substantial amount $\mu$ and then define the substantial density by

$\rho(x)=\lim _{\epsilon \rightarrow 0} \frac{\mu\left(B_{\epsilon}(x)\right)}{\lambda\left(B_{\epsilon}(x)\right)}$,

where $B_{\epsilon}(x)$ is an $\epsilon$-ball centered at $x$, and $\lambda$ is the Lebesgue measure on $\mathcal{X}$. Substantial density and substantial amount are two sides of the same coin, and this coin represents the ability to quantify the amount of substance contained in regions of physical space.

When for two regions $X$ and $Y$ we have $\mu(X)=c \cdot \mu(Y)$, then this means that there is $c$ times more substance contained within $X$ than within $Y$. It is exactly this ability to numerically measure and compare the amount of substance contained in different regions in space, which justifies the physical notion of "substance". If different heaps of a substance could not be measured and compared with respect to their quantity, the term "substance" would be inadequate. Only, we have to refrain from the idea that a substance must necessarily be composed of discrete entities such as particles. It is conceivable, and mathematically describable, that a substance is truly continuous and can still be measured with respect to its quantity. 
Note that we are not talking about the number of mathematical points in a set, which would be given by the cardinality of the set. The cardinality, or cardinal number, is an abstract mathematical concept introduced by Georg Cantor, the inventor of set theory. Two sets have the same cardinality exactly if there is a one-to-one mapping between the elements of both sets. For finite sets, the cardinality equals the number of elements in the set. For infinite sets, the cardinality is a so-called transfinite number, denoted by $\aleph_{0}, \aleph_{1}, \ldots$, so that the set $\mathbb{N}$ is defined to have the smallest transfinite cardinality $\aleph_{0}$, which is also denoted as countably infinite. The cardinality $\mathfrak{c}=2^{\aleph_{0}}$ of the continuum can be proven to be bigger than $\aleph_{0}$ (in the sense that there is no one-to-one mapping), but its actual value is logically independent from the axioms of ZFC set theory. The value of $\mathfrak{c}$ depends on whether one accepts the continuum hypothesis or not, which postulates that $\mathfrak{c}=\aleph_{1}$. The cardinality of sets is a profound and fruitful concept exploring the depths of mathematical logic, but it has few to do with physical considerations. The amount $\mu$ of a substance contained in a given region in space is not to be confused with the cardinal number of mathematical points within that region. While the cardinality is the set theoretic number of elements in a given set, $\mu$ is the integrated spatial density of a substance in a given region in space, and as such it is not a property of space itself, hence not an a priori measure, but rather a property of the physical substance being distributed in space, hence an a posteriori measure. A typical a priori measure of space would be the spatial volume as provided by the Lebesgue measure. In contrast, the substantial amount $\mu$ is an a posteriori measure that captures the contingent spatial distribution of a physical substance under consideration. Different measures $\mu$ would correspond to different spatial distributions of the substance. Yet, to speak of a substance $S$ being distributed in space is just to speak of a particular measure $\mu$ on that space, so that $\mu(X)$ yields the substantial amount of $S$ contained within the region $X$. The substantial density and accordingly the substantial amount are physical properties of a substance in the same manner that, say, energy is a physical property of a system. Energy has no mathematical meaning; it only has a mathematical form. Same with the substantial density and the substantial amount: they have a mathematical form, and this form has mathematical properties. But the meaning of these notions is physical.

The substantial amount can be regarded as a straight generalization of the number of particles of a discrete substance, which can be seen by considering a substantial density of the form

$\rho(x) \sim \sum_{n=1}^{N} \delta\left(x-x_{n}\right)$,

for some finite number $N$ of point-like particles located at positions $x_{n}$. Then, the substantial amount of stuff concentrated within a finite region $X$ is proportional to the number of particles contained within that region,

$\mu(X) \sim \int_{X} d^{3} x \sum_{n=1}^{N} \delta\left(x-x_{n}\right)=\left|\left\{n \mid x_{n} \in X\right\}\right|$.

Thus, discrete substances are really just a special case where the substantial density has singularities, which form discontinuities of the substantial amount, and these discontinuities are identifiable as particles of the substance.

If we consider the trajectories across configuration space corresponding to different worlds as physically existing, they constitute a continuous substance, and we can apply the above concepts, so that $\rho$ now measures the density of trajectories in configuration space, and $\mu$ measures the amount of trajectories contained within a given region in configuration space.

\subsection{Re-thinking Laplace}

In his famous "Essay on Probability", Laplace [26,27] introduced probability as the degree of certainty, or credence, to obtain a desired outcome from a finite set of possibilities. More precisely, if $A$ is the set of favorable outcomes and $\Omega$ is the set of possible outcomes, then the probability to obtain a favorable outcome is defined as

$P(A)=\frac{|A|}{|\Omega|}$, 
where $|\cdot|$ counts the number of elements. There are numerous ways to justify Laplace's rule, but most of them are circular. For example, deriving Laplace's rule from an assumption of uniform probability on the set $\Omega$ only shows that Laplace's rule is consistent with probability theory. The very notion of probability itself, though, cannot be derived from probability assumptions. What Laplace had in mind was to postulate a quantity called "probability" that applies to a certain kind of situation where we have to quantify our degree of certainty that something is the case. Whenever we are completely indifferent which one of a given finite set of possibilities is actually realized, then we ought to apply Laplace's rule. This conception of probability is an epistemic conception, that is, it relates to the knowledge of an observer. Two observers with distinct states of knowledge may attribute distinct probability distributions to the same set of possibilities. There is no objective probability distribution, so probability is nothing existing "out there" (cf. [7]). I adhere to this conception of subjective probability, and I think it is all one needs to also understand the probabilistic aspect of quantum mechanics.

The direct translation of Laplace's rule to the situation of an observer in an objectively existing multiplicity of worlds would be to take $\Omega$ as the set of all worlds, because each world may possibly be the world of the observer. However, in the here-proposed theory there is a continuum of worlds, so there is no such thing as the "number" of worlds, and the ratio (10) would be ill-defined. We, therefore, have to generalize Laplace's rule to infinite sets, but how may this be reasonably accomplished?

Laplace did not give any reason why the probability should be equal to the ratio (10); he simply defined it so. So let us fancy a justification. If $A$ and $B$ are two sets of possibilities, and there are $c$ times more possibilities in $A$ than there are in $B$, then it should be $c$ times more probable that the actually realized possibility lies in $A$ than that it lies in $B$. Consequently, if there is a plausible measure $\mu$ on the set $\Omega$ of possibilities, so that $\mu(A)=c \cdot \mu(B)$ means that $A$ contains $c$ times more possibilities than $B$, then we should expect that

$$
\frac{P(A)}{P(B)}=\frac{\mu(A)}{\mu(B)}
$$

The final step is then a convenient normalization of probability, $P(\Omega)=1$, so that we obtain

$$
P(A)=\frac{\mu(A)}{\mu(\Omega)} \text {. }
$$

The advantage of these considerations is that they do not rely upon the sets to be finite. Whenever there is a reasonable concept of an "amount" of possibilities provided by a measure $\mu$ on the set of all possibilities $\Omega$, then there is a related probability measure $P$ on $\Omega$. Of course, for different measures $\mu$ we would obtain different probability measures $P$, so there needs to be an independent justification why a certain measure $\mu$ is the relevant one. Such independent justification cannot be provided by mathematics alone but has to be a physical justification obtained within the framework of a physical theory. The important thing to note is that the physical theory does not have to provide the probability concept itself. These are independent reasonings. The physical theory yields the justification for a measure $\mu$, and then the probability considerations apply independently. In the finite case the measure $\mu$ is naturally provided by the number of possibilities, but what is the relevant measure in the case of uncountably many worlds?

In Sect. 2.3 we have seen that there is a one-to-one correspondence between worlds and trajectories in configuration space. Thus, for every world $w$ there is a unique trajectory $\gamma$ in configuration space, and this trajectory is parameterized by the time parameter $t$. The trajectory point $q=\gamma_{t}$ is then the configuration of the universe in the world corresponding to $\gamma$ at time $t$. Different trajectories are not allowed to cross each other, because otherwise there would be more than one world for an individual configuration (at the crossing point of the trajectories), which is forbidden by the uniqueness relations (1) and (2). A system of non-crossing trajectories is a deterministic system, which means that each trajectory is completely determined by any one of its points (see $[15,48]$, for thorough discussions on the role of determinism in modern physics). We may represent the entire collection of trajectories $\Gamma=\{\gamma\}$ by a single trajectory function $\xi$, so that $\gamma_{t}=\xi_{t}\left(\gamma_{0}\right)$. Note that up to now we have nowhere specified that the trajectories have to obey a differential equation. They may be (so far) non-differentiable, yet even discontinuous, but still they would be deterministic in the sense that they would be completely determined by any one of their points. 
Let the substantial density of trajectories crossing an infinitesimal region centered at $q$ be given by some density function $\rho_{t}$. Then the substantial amount of trajectories crossing some finite region $Q$ at time $t$ reads

$\mu_{t}(Q)=\int_{Q} \mathrm{~d} q \rho_{t}(q)$

If for two regions $X$ and $Y$ we have $\mu(X)=c \cdot \mu(Y)$, then this means that there are $c$ times more trajectories crossing $X$ than there are trajectories crossing $Y$, where "more" is to be understood in a physical sense. Mathematically, there are exactly as many trajectories crossing $X$ than there are trajectories crossing $Y$, namely $\mathfrak{c}=2^{\aleph_{0}}$. But the substantial amount is not about mathematical entities but rather about a physical substance that is described mathematically. There is $c$ times more substance in $X$ than in $Y$, and this particular substance is composed out of trajectories. Now, since each trajectory corresponds to exactly one world, $\mu_{t}$ measures the substantial amount of worlds whose trajectories cross the region $Q$ at time $t$. Each world corresponds to a possibility, namely the possibility that this world is our world. When a region $X$ contains $c$ times more worlds than another region $Y$, then according to our probability considerations above it should be $c$ times more probable that our world is contained in $X$ than in $Y$, so

$\frac{P_{t}(X)}{P_{t}(Y)}=\frac{\mu_{t}(X)}{\mu_{t}(Y)}$.

Since our world is with certainty at any time somewhere in $\mathcal{Q}$, so $P_{t}(\mathcal{Q})=1$, it follows that

$P_{t}(Q)=\frac{\mu_{t}(Q)}{\mu_{t}(\mathcal{Q})}$

Say, Joe is at time $t$ about to measure an observable $A$ that obtains values $a_{1}, a_{2}, \ldots$ in mutually disjoint and exhaustive sets of worlds $W_{1}, W_{2}, \ldots$, that is, $W_{i} \cap W_{j}=\emptyset$ for $i \neq j$, and $\bigcup_{i} W_{i}=\mathcal{W}$. Furthermore, say that the trajectories in these worlds are at time $t$ crossing the respective regions $Q_{1}, Q_{2}, \ldots$ in configuration space. Since there is a one-to-one correspondence between worlds and trajectories, and since there is a one-to-one correspondence between trajectories and their points at time $t$, for $t$ being arbitrarily given, the regions $Q_{1}, Q_{2}, \ldots$ are also mutually disjoint and exhaustive. Then, the probability $p_{k}$ that Joe will find himself in a world where $A$ obtains the value $a_{k}$ is given by

$p_{k}=P_{t}\left(Q_{k}\right)$.

Since $P_{t}$ is a probability measure on $\mathcal{Q}$ and since the regions $Q_{1}, Q_{2}, \ldots$ are mutually disjoint and exhaustive, the numbers $p_{k}$ fulfill the requirements of a probability distribution, that is $p_{k} \geq 0$, and $\sum_{k} p_{k}=1$.

In the next section we have to establish a link between the substantial density $\rho_{t}$ of trajectories in configuration space and the wavefunction $\Psi_{t}$. This link cannot be provided other than by postulating that the substantial density of trajectories crossing an infinitesimal region centered at the point $q \in \mathcal{Q}$ is given by $\rho_{t}(q)=\left|\Psi_{t}(q)\right|^{2}$. This postulate complements another central postulate, which links the course of each trajectory to the wavefunction via the Bohm equation (33). These two postulates give the wavefunction a physical meaning, namely that of a generating function for a continuous substance formed by a bundle of trajectories distributed in configuration space. Our world is part of this substance, traveling along its trajectory through configuration space beneath uncountably many other worlds.

\section{The wavefunction}

A central element of quantum theory is the wavefunction $\Psi_{t}$, and a great challenge for any interpretation of quantum mechanics is to give a physical meaning to the wavefunction. Does it represent a physical entity itself or is it just a mathematical tool to calculate probabilities? No doubt the wavefunction is physically significant, as it appears in the fundamental equations from where observable values are derived. But beyond its physical significance, the physical meaning of the wavefunction is subject to longstanding controversial debates. In Bohmian mechanics, the wavefunction represents a physical entity, also called the pilot wave or the guiding field, and it is conceived of as a physical field in configuration space that guides all particles in the universe along their trajectories. In Everettian 
mechanics, the wavefunction is also a physically existing entity, and, moreover, the wavefunction is all there is in the universe. Particles and macroscopic objects only appear as patterns formed by the wavefunction in the course of its temporal evolution (cf. [55]). Here, I wish to propose a different picture. The wavefunction is, in this picture, not a physically existing entity itself, but rather an abstract mathematical tool to determine the form of a physically existing continuum of trajectories of varying density in configuration space, called the world continuum, which is identified with the history of the universe. The wavefunction thus has an ontic meaning rather than an epistemic one: it represents a compact and complete mathematical representation of the objectively existing history of the universe, and not of our state of knowledge about the universe. In the following we shall get some more into detail.

\subsection{Configuration space}

For systems of more than one particle, the wavefunction is a function not in the three-dimensional space but in the $3 N$-dimensional configuration space. This raw mathematical fact is a serious obstacle towards a straightforward physical interpretation of the wavefunction. For if the wavefunction is taken to be (or to represent) a physically existing entity, then should not the configuration space be considered the real space, instead of the 3D space? And even if the wavefunction is taken to be just a mathematical construction, is the configuration space not still a more adequate representation of physical reality than the 3D space?

Consider a single particle. According to the world continuum theory, the particle is in every world and at every time located at an exact point in 3D space. Let us concentrate on one individual world $w$ having a trajectory $\boldsymbol{\gamma}$. At each time $t$ the particle is located at a certain position $\boldsymbol{q}=\boldsymbol{\gamma}_{t}$. As all time points are considered to be equally real, the particle in world $w$ is physically represented not by a single point in 3D space but rather by its entire trajectory $\boldsymbol{\gamma}$, which is a one-dimensional object in space and time, it is a curve and not a point of dimension zero. As all worlds are taken to be equally real as well, the particle is altogether physically represented by a continuous bundle of trajectories from the set $\Gamma=\{\boldsymbol{\gamma}\}$. In the same way as the particle's trajectory in one world is composed out of continuously many points, one point per time point $t$, the trajectory bundle is composed out of continuously many trajectories, one trajectory per world.

Now consider $N$ particles. In world $w$ and at time $t$ these particles are located each at an exact position in 3D space. Say, particle $n$ is located at time $t$ at the position $\boldsymbol{q}_{n}=\boldsymbol{\gamma}_{n, t}$, with $n=1, \ldots, N$. The mathematical representation of the location of the particles is a list of their three-dimensional positions, $q \equiv\left(\boldsymbol{q}_{1}, \ldots, \boldsymbol{q}_{N}\right)$. This list of positions can mathematically be interpreted as a vector in the $3 N$-dimensional space $\mathbb{R}^{3 N}$, which is commonly taken as the configuration space. However, is the configuration space just a mathematical construction or is it a physically existing entity? A trajectory in configuration space can be interpreted as representing $N$ particles moving through the real 3D space, where "moving" just means that at different time points there are different positions taken by the particles. These $N$ particles form a system, which is the mereological sum of the particles. Thus, a trajectory through configuration space can alternatively be interpreted as representing one single object, the universe, moving through configuration space. These two different interpretations are equivalent, but one interpretation, the one that views the universe as a unified object, better reflects the phenomenon of quantum nonlocality. Why so? In a local theory, each individual particle trajectory $\boldsymbol{\gamma}_{n}$ must be a solution of a hyperbolic differential equation, and indeed classical mechanics is such a local theory (ignoring here the delicate issue of the gravitational potential). However, quantum mechanics is a not a local theory in this respect. The trajectories of individual particles are not solutions to hyperbolic differential equations. However, the trajectory $\gamma$ of the entire universe is a solution to a hyperbolic differential equation, namely the Bohm equation (20). The trajectories of individual particles depend on the wavefunction, and the wavefunction is a function on configuration space and not on 3D space. As a consequence, the movement of an individual particle depends on the instantaneous positions of the other particles, however distant in space they are. It is one of the most puzzling features of quantum mechanics, though, that such nonlocal interdependency between particles cannot be exploited for superluminal signaling, as has been proven in the context of Bohmian mechanics by Valentini [50]. Intriguingly, thus, quantum mechanics is a nonlocal theory, on account of the mathematical definition of locality, but it is a local theory with respect to 
Einstein locality, which amounts to the assertion that superluminal signaling is impossible. In other words, Nature as described by quantum mechanics is epistemically local, that is, it appears to observers as local, in the sense that they cannot communicate or gain information in a nonlocal manner, but ontologically, with respect to sheer existence, Nature is nonlocal. This ontological nonlinearity is not just a philosophical subtlety, but it has observable physical implications, namely those phenomena usually termed as being typically quantum, such as quantum interference, tunneling, teleportation, and the like. To better reflect the ontological nonlocality of Nature, I consider it more adequate to view the universe as one unified entity extending in time and configuration space, and not in time and 3D space.

Now, there is a widely ignored problem with interpreting the configuration space $\mathbb{R}^{3 N}$ as the physical space, and it is that the three spatial dimensions of each particle are lumped together into one column vector with $3 \mathrm{~N}$ components. While for most physicists this might appear rather unproblematic, it makes the theory vulnerable against a subtle but profound criticism put forward by Monton [31]. As the author writes, the problem is essentially "...that nowhere in the $3 \mathrm{~N}$-dimensional space is it specified which dimensions correspond to which particles", which leads the author to conclude that "the wave function ontology is an undesirable ontology for quantum mechanics". Monton's criticism applies to those quantum mechanical theories that entail wavefunction realism in some way, such as Bohmian mechanics and Everettian mechanics. It would also apply to the here-proposed theory if one favors the configuration space as the real space to pronounce quantum nonlinearity as a natural phenomenon.

Monton's criticism can straightforwardly be addressed by separating spatial dimensions and particle associations into the rank-two tensor space $\mathbb{R}^{3 \times N}$, which in the following will be referred to as the configuration space $\mathcal{Q}$, instead of $\mathbb{R}^{3 N}$. While a point $\boldsymbol{q}=(x, y, z)^{T}$ is a column vector in the space $\mathbb{R}^{3}$, a point $q=\left(\boldsymbol{q}_{1}, \ldots, \boldsymbol{q}_{N}\right)$ is a rank-two tensor in the space $\mathbb{R}^{3 \times N}$, that is, a matrix

$q=\left(\begin{array}{l}x_{1} \cdots x_{N} \\ y_{1} \cdots y_{N} \\ z_{1} \cdots z_{N}\end{array}\right)$.

Since any tensor space is also a linear space, and in that sense still a mathematical vector space that can be endowed with an inner product just like ordinary rank-one vector spaces, one does not sacrifice mathematical structure. Also, there is no difficulty in defining wavefunctions $\Psi$, $\Phi$ on the tensor space $\mathbb{R}^{3 \times N}$ instead of $\mathbb{R}^{3 N}$, so that the Hilbert inner product can be defined as

$$
\langle\Psi \mid \Phi\rangle:=\int \mathrm{d} q \Psi^{*} \Phi
$$

which is a shorthand notation of

$$
\int \mathrm{d} q \Psi^{*} \Phi \equiv \int_{\mathbb{R}^{3}} \mathrm{~d}^{3} q_{1} \cdots \int_{\mathbb{R}^{3}} \mathrm{~d}^{3} q_{N} \Psi^{*}\left(\boldsymbol{q}_{1}, \ldots, \boldsymbol{q}_{k}\right) \Phi\left(\boldsymbol{q}_{1}, \ldots, \boldsymbol{q}_{k}\right) .
$$

A trajectory $\gamma$ represents the movement of $N$ particles through 3D space in time. A point $q=\gamma_{t}$ on the trajectory represents the configuration of the universe at time $t$, and the $(i, j)$-th component $q_{i j}=\left(\boldsymbol{q}_{j}\right)_{i}$ corresponds to the $i$-th spatial component of the position of the $j$-th particle at $t$. The confusion of spatial dimensions and particle associations criticized by Monton does not arise, as it is clearly specified which spatial dimensions correspond to which particles.

\subsection{World continuum}

According to the view so far developed, the universe as a whole is physically represented by a continuous bundle $\Gamma=\{\gamma\}$ of trajectories in configuration space, where each trajectory $\gamma \equiv\left(\boldsymbol{\gamma}_{1}, \ldots, \boldsymbol{\gamma}_{N}\right)$ represents the movement of all particles in the universe. The entire trajectory bundle $\Gamma$ can be represented by a single trajectory function $\xi=\xi_{t}(q)$, so that for each $\gamma \in \Gamma$ we have $\gamma_{t}=\xi_{t}\left(\gamma_{0}\right)$. In order for the trajectory bundle $\Gamma$ to be considered as a continuous substance, the world continuum, there is one more feature to be provided: the substantial density of the 
trajectories in configuration space. At any given time $t$, the trajectories may in some regions of configuration space be more densely packed than in other regions, and this feature is governed by a time-dependent density $\rho=\rho_{t}(q)$ on configuration space, which is linked to a the measure $\mu=\mu_{t}(Q)$ on configuration space via (13). So, the world continuum is uniquely represented by the tuple $\Upsilon=(\rho, \xi)$. The world continuum, so I propose, is an adequate and complete picture of the history of the physically existing universe. The wavefunction $\Psi$ is not a physically existing entity itself but rather an abstract generating function that determines the form of the world continuum. In order for this to make sense, the wavefunction $\Psi$ must contain all information necessary to uniquely determine the form of the world continuum, that is, the course of each individual trajectory as well as the density of the trajectories in configuration space. The course of each individual trajectory is determined by the guiding equation of Bohmian mechanics, which in the context of the here-proposed theory should be rather called a trajectory equation, or simply the Bohm equation. That is, each trajectory $\gamma$ is a solution of the first-order differential equation

$\frac{\mathrm{d}}{\mathrm{d} t} \gamma_{t}=\frac{j_{t}}{\rho_{t}}$

where the vector field

$j_{t} \equiv\left(\boldsymbol{j}_{t, 1}, \ldots, \boldsymbol{j}_{t, N}\right)$

is called the world flow, defined by

$j_{t, n}=\frac{\hbar}{2 m_{n} i}\left(\Psi_{t}^{*} \nabla_{n} \Psi_{t}-\Psi_{t} \nabla_{n} \Psi_{t}^{*}\right)$

and where the scalar field

$\rho_{t}=\left|\Psi_{t}\right|^{2}$

is called the world density. The set of all solutions yields the set $\Gamma=\{\gamma\}$, and each trajectory $\gamma \in \Gamma$ corresponds to exactly one world $w \in \mathcal{W}$. Since the set of solutions is uncountable, so is the set of worlds.

Now, why is $\rho$ called the world density and $j$ called the world flow? Because if the wavefunction $\Psi$ obeys the Schrödinger equation

$i \hbar \frac{\partial}{\partial t} \Psi_{t}=\hat{H} \Psi_{t}$

for some given Hamiltonian $\hat{H}$, then it can easily be shown that the functions $\rho$ and $j$ fulfill the continuity equation

$$
\frac{\partial}{\partial t} \rho_{t}+\nabla \cdot j_{t}=0
$$

where $\nabla \equiv\left(\nabla_{1}, \ldots, \nabla_{N}\right)$ is the nabla operator on configuration space, and where the scalar product of two vectors $a, b \in \mathcal{Q}$ is defined as $a \cdot b:=\sum_{n=1}^{N} \boldsymbol{a}_{n} \cdot \boldsymbol{b}_{n}$. As Madelung [29] already pointed out, the functions $\rho$ and $j$ describe a locally conserved compressible fluid in configuration space. In fact, Bohmian trajectories are nothing but the pathlines, or integral lines, of this fluid. However, Madelung could not provide a consistent physical interpretation of this mathematical fact, so the hydrodynamical interpretation of quantum mechanics was abandoned. Recently, the hydrodynamic interpretation experienced a renaissance, and it was shown that the wavefunction can be completely removed from the theory, leaving only trajectories as the physically existing objects from where all observable values can be calculated $[23,35,39]$. However, these approaches leave it open as to how the fluid is interpreted physically. Holland [23] remarks that their model is particularly suited to interpret the fluid as being composed of a continuum of "probability elements", in line with the standard view of interpreting the continuity equation as describing a "probability fluid". Whatever probability really is, it is certainly not a material entity. In the interpretation of the here-proposed theory, in contrast, the fluid is interpreted as a material entity, the world continuum, and the fluid elements flowing through configuration space are worlds. ${ }^{2}$

\footnotetext{
2 To say that a world flows through configuration space is another way of saying that the configuration of the universe in that world is parameterized by time.
} 
Under the usual assumption that the wavefunction vanishes rapidly enough at infinity, one may integrate the continuity equation over the configuration space, which yields

$$
\frac{\partial}{\partial t} \int \mathrm{d} q \rho_{t}=0
$$

Thus the integral of the world density $\rho_{t}$ over the entire configuration space is constant in time and we have

$$
\int \mathrm{d} q \rho_{t}=\int \mathrm{d} q \rho_{t^{\prime}}=\mu_{0},
$$

for any two time points $t, t^{\prime}$. We then define the world amount by

$\mu_{t}(Q)=\int_{Q} \mathrm{~d} q \rho_{t}$,

so the total amount of worlds $\mu_{t}(\mathcal{Q})$ is constant in time and equal to $\mu_{0}$. Physically, the constancy of $\mu_{t}(\mathcal{Q})$ means that there are no worlds being destroyed or created during the evolution of the universe. As each world corresponds to a really existing trajectory in configuration space, the density function $\rho_{t}$ is not a probability density but the substantial density of really existing trajectories. That is, the integral of $\rho_{t}$ over some region $Q$ in configuration space does not yield a probability, but rather the substantial amount $\mu_{t}(Q)$ of trajectories crossing the region $Q$ at time $t$. Due to local conservation of $\mu$, as expressed by the continuity equation (25), the trajectories have no beginnings and no endings, and neither do they split nor converge. This stands in contrast to Everettian mechanics with its ontology of splitting worlds. As we have seen earlier, one can derive from $\mu_{t}$ the epistemic probability $P_{t}$ corresponding to the ignorance of an observer about which world it is that he or she lives in. So, importantly, probability is in the world continuum theory a derived concept rather than a fundamental one. In a continuum of worlds distributed with a certain density $\rho_{t}$, the subjective probability to find oneself within a particular world must be given by (15), as a result of probability considerations external to, and independent from, the physical theory itself.

The wavefunction as a generating function of the world continuum contains two redundant parameters, which is the global scale and the global phase. This is because the Bohm equation (20) is symmetric under the transformation $\Psi_{t} \mapsto r e^{i \phi} \Psi_{t}$ for $r>0$ and $0 \leq \phi<2 \pi$, so the trajectories do not depend on the global scale and the global phase. The world density (23) is symmetric with respect to the global phase $\phi$ but it scales quadratically with the global scale $r$. However, a global scaling of the world density bears no physical significance, because it leaves the relative proportions between different world amounts untouched. A region $X$ will still contain $c$ times more worlds than another region $Y$, irrespective of the global scaling factor.

Besides the global scaling and the global phase there are no further redundancies, as can be seen by writing the wavefunction in polar decomposition $\Psi_{t}=R_{t} e^{i S_{t}}$, so that the Bohm equation (20) and the world density (23), respectively, become

$\frac{\mathrm{d}}{\mathrm{d} t} \boldsymbol{\gamma}_{n}=\frac{\hbar}{m_{n}} \nabla_{n} S_{t}$

and

$\rho_{t}(q)=R_{t}^{2}$.

Thus, the phase $S_{t}$ of the wavefunction generates the trajectory bundle $\Gamma=\{\gamma\}$ represented by the trajectory function $\xi$, and the amplitude $R_{t}$ generates the trajectory density $\rho$. Altogether the wavefunction can be regarded as a generating function of the tuple $\Upsilon=(\rho, \xi)$ that mathematically represents the world continuum (Fig. 2). The wavefunction (not the projective ray in Hilbert space, of which the wavefunction is a representative) contains slightly more information than $\Upsilon$. If from both the wavefunction and from the world continuum $\Upsilon$ all observable values can be calculated, then the world continuum is a slightly less redundant representation of physical reality than the wavefunction. The world continuum is, however, just as informative as a projective ray in Hilbert space, which is an equivalence class of wavefunctions differing only by their global scale and phase. From the perspective of Occam's razor, thus, the world continuum theory is just as ontologically demanding as any theory that entails wavefunction 


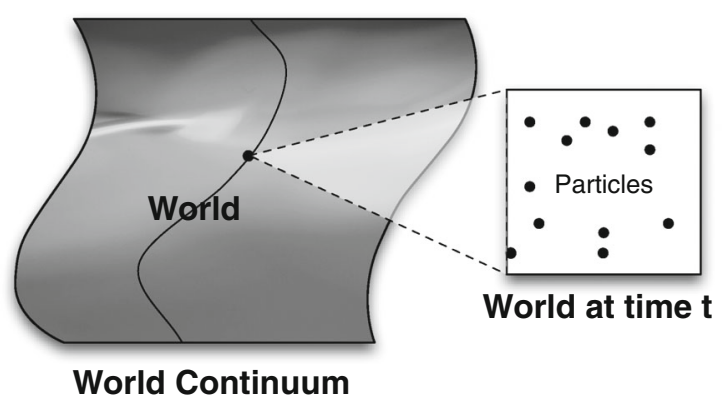

Fig. 2 Schematic illustration of the ontology of the proposed theory. The world continuum is a continuous bundle of trajectories in configuration space, with each trajectory uniquely corresponding to a world. The density of the trajectories in configuration space is defined by a density function $\rho_{t}=\left|\Psi_{t}\right|^{2}$. Each trajectory $\gamma$ represents the movement of $N$ point-like particles through 3D space and is a solution of the Bohm equation $d \gamma_{t} / d t=j_{t} / \rho_{t}$, where the flow $j_{t}$ is also derived from the wavefunction. From the density $\rho_{t}$, the Born rule can be derived as the subjective probability of finding oneself within a particular world. The wavefunction itself is not a physically existing entity but merely serves as a generating function for the density and the flow that both determine the form of the physically existing world continuum

realism. This includes Bohmian mechanics as well as Everettian mechanics. As for Bohmian mechanics, the ontological costs are somewhat higher, because in addition to a physically existing wavefunction there are $N$ physically existing point-like particles. As for the Copenhagen interpretation, for that matter, the wavefunction is usually not taken as a physically existing entity but rather as a mathematical tool to calculate probabilities. However, the Copenhagen interpretation issues of its own, most of which are comprised under the term "measurement problem"), but these are not under discussion here.

\section{Foundations of the theory}

From the preceding considerations we shall now distill a minimal set of postulates that generates the theory. For the sake of simplicity, we will stay with the case of spin-free particles. Spin can be included in the same manner as in Bohmian mechanics by promoting the scalar wavefunction $\Psi=\Psi_{t}(q)$ to a spinor wavefunction $\vec{\Psi}=\Psi_{t}^{\sigma_{1} \ldots \sigma_{N}}(q)$, and by extending the Hamiltonian to include spin interaction (cf. [34]).

\subsection{Axiomatics}

Postulate 1 The physical history of a closed system of $N$ spin-free particles is completely described by a wavefunction $\Psi=\Psi_{t}(q)$. Each time-instance $\Psi_{t}$ is a vector in the Hilbert space $\mathcal{H}=L^{2}\left(\mathbb{R}^{3 \times N}\right)$, and is called the state of the system at time $t$. The wavefunction $\Psi$ is a solution of the Schrödinger equation

$i \frac{\mathrm{d}}{\mathrm{d} t} \Psi_{t}=\hat{H} \Psi_{t}$,

where $\hat{H}$ is the Hamiltonian of the system, given by

$\hat{H}=\sum_{n=1}^{N} \frac{\hat{\boldsymbol{p}}_{n}^{2}}{2 m_{n}}+V(\hat{q})$,

where $\hat{\boldsymbol{p}}_{n}=\frac{\hbar}{i} \nabla_{n}$ is the momentum operator of the $n$-th particle, and $\hat{\boldsymbol{q}}_{n} \Psi(q)=\boldsymbol{q}_{n} \Psi(q)$ is the position operator of the $n$-th particle, so that $\hat{q}=\left(\hat{\boldsymbol{q}}_{1}, \ldots, \hat{\boldsymbol{q}}_{N}\right)$ is the configuration operator, and the operator-valued function $V(\hat{q})$ is the potential energy of the system. 
Postulate 2 The physical history of the system in a specific world is completely described by a trajectory $\gamma=\gamma_{t}(q)$, which is a solution of the Bohm equation

$\frac{\mathrm{d}}{\mathrm{d} t} \gamma_{t}=\frac{j_{t}}{\rho_{t}}$,

where $j_{t}=\left(\boldsymbol{j}_{1, t}, \ldots, \boldsymbol{j}_{N, t}\right)$ is defined by

$j_{n, t}=\frac{\hbar}{2 m_{n} i}\left(\Psi_{t}^{*} \nabla_{n} \Psi_{t}-\Psi_{t} \nabla_{n} \Psi_{t}^{*}\right)$

and where $\rho_{t}$ is defined by

$\rho_{t}=\left|\Psi_{t}\right|^{2}$.

Each solution $\gamma$ of the Bohm equation (33) is a world-instance of the system, and the world-time-instance $\gamma_{t}$ is the configuration of the system at time $t$ in the world corresponding to $\gamma$.

Postulate 3 The trajectories form a continuous substance in configuration space, with the function $\rho=\rho_{t}(q)$ representing the substantial density of trajectories. Therefore, the substantial amount of trajectories crossing a finite region $Q$ in configuration space at time $t$ is given by

$\mu_{t}(Q)=\int_{Q} \mathrm{~d} q \rho_{t}(q)$,

so that for two regions $X$ and $Y$ in configuration space with $\mu_{t}(X)=c \cdot \mu_{t}(Y)$ this means that there are $c$ times more trajectories contained within $X$ than there are contained within $Y$. As each trajectory corresponds to exactly one world, $\rho$ is also called the world density and $\mu_{t}$ is also called the world amount.

\subsection{Remarks}

Remark 1 The theory provides the following picture of reality, for a given closed system $S$ that can also represent the entire universe:

$$
\begin{aligned}
\text { wavefunction } \Psi & \leftrightarrow \text { physical history of } S \\
\text { state } \Psi_{t} & \leftrightarrow \text { time-instance of } S \\
\text { trajectory } \gamma & \leftrightarrow \text { world-instance of } S \\
\text { configuration } \gamma_{t} & \leftrightarrow \text { world-time-instance of } S
\end{aligned}
$$

Remark 2 Since (31) and (33) are first-order hyperbolic equations, they have a unique solution for every valid initial condition. More precisely, for every well-behaved initial quantum state $\Psi_{0} \in \mathcal{H}$ at time $t=0$ there is a unique wavefunction $\Psi$ that is obtained by applying the unitary time evolution operator

$\hat{U}(t)=e^{-i \hat{H} t}$,

so that

$\Psi_{t}=\hat{U}(t) \Psi_{0}$.

Similarly, for every initial configuration $\gamma_{0} \in \mathcal{Q}$ for which $\rho_{0}\left(\gamma_{0}\right)>0$, there is a unique trajectory $\gamma$ obtained by applying the trajectory function

$\xi_{t}=\int_{0}^{t} \mathrm{~d} t^{\prime} \frac{j_{t^{\prime}}}{\rho_{t^{\prime}}}$ 
so that

$\gamma_{t}=\xi_{t}\left(\gamma_{0}\right)$.

Hence, in each world there is a concrete path through space that the particles take, and which is determined by the trajectory function $\xi_{t}$ applied to the initial configuration $\gamma_{0}$ at $t=0$. The path $\boldsymbol{x}_{n}(t)$ of an individual particle $n$ can be extracted from the trajectory function $\xi_{t}=\left(\xi_{1, t}, \ldots, \boldsymbol{\xi}_{N, t}\right)$ by fetching the components corresponding to that particle, so that $\boldsymbol{x}_{n}(t)=\xi_{n, t}\left(\gamma_{0}\right)$. Being a time-dependent function on the configuration space, the trajectory function $\xi_{t}$ can also be regarded as a dynamical vector field in configuration space, assigning each point $q \in \mathcal{Q}$ its time-evolved counterpart $q_{t}=\xi_{t}(q)$. Using the trajectory function, the time-evolved world density can also be written as

$\rho_{t}(q)=\int \mathrm{d} q^{\prime} \delta\left(q-\xi_{t}\left(q^{\prime}\right)\right) \rho_{0}\left(q^{\prime}\right)$

Remark 3 Due to the Schrödinger dynamics, the functions $\rho$ and $j$ can be shown to obey the continuity equation

$\frac{\mathrm{d}}{\mathrm{d} t} \rho_{t}+\nabla \cdot j_{t}=0$

so that $j$ takes the role of a trajectory current. The substantial flow of trajectories crossing at time $t$ a $(2 \times N)$ dimensional directed submanifold $F=F_{1} \times \cdots \times F_{N}$, where each $F_{n}$ is a two-dimensional directed manifold in $\mathbb{R}^{3}$, is given by

$v_{t}(F)=\int_{F} \mathrm{~d} F \cdot j_{t}$,

where $\mathrm{d} F=\left(\mathrm{d} \boldsymbol{F}_{1}, \ldots, \mathrm{d} \boldsymbol{F}_{N}\right)$ is the infinitesimal normal vector element on the directed manifold $F$. As each trajectory corresponds to exactly one world, $j$ is also called the world current. Negative values for the substantial flow indicate that there are more worlds with particles crossing the directed manifold $F$ in reverse direction.

\subsection{Measurement}

In standard quantum mechanics, measurement is an additional concept different from that of ordinary Schrödinger evolution. In the world continuum theory, just like in Bohmian mechanics and Everettian mechanics, measurement is a specially designed but otherwise ordinary physical process that involves a short and strong interaction between the system of interest and a macroscopic measurement device involving a large number of particles. The measurement process is modeled in the same way as it is done in Bohmian mechanics, and in a similar way as in Everettian mechanics, except that the "pointer basis problem" of Everettian mechanics does not show up, because we can adopt from Bohmian mechanics the concept of pointer states as spatially separated wave packets. The key idea is that different sets of pointer configurations correspond to different measurement outcomes, so when a pointer configuration lies within some region $Z_{a}$ of the pointer configuration space, then this is taken to indicate the measurement outcome " $a$ ". In contrast to Bohmian mechanics, though, there is no "true" configuration, but rather all configurations equally exist in different worlds.

Consider a collection of mutually disjoint compact regions $Z_{a}$ in the pointer configuration space $\mathcal{Q}_{M}$, so that for $a \neq a^{\prime}$ we have

$Z_{a} \cap Z_{a^{\prime}}=\emptyset$.

Furthermore, let there be a corresponding collection of pointer states $\eta_{a}$, which have almost all of their support within the corresponding regions $Z_{a}$, so

$$
\int_{Z_{a}} \mathrm{~d} z\left|\eta_{a}(z)\right|^{2} \approx 1
$$


Let us call $Z_{a}$ an effective support of $\eta_{a}$. From (48) and (49), and since the pointer states are normalized to unity, it follows that the pointer states have almost no spatial overlap, so for $a \neq a^{\prime}$ we have

$\eta_{a}(z) \eta_{a^{\prime}}(z) \approx 0$,

so the pointer states are quasi-orthogonal to each other. In the idealized case where the overlap of the pointer states is exactly zero, the pointer states are perfectly orthogonal to each other. However, this would require each pointer state to have a compact support in configuration space, and such wavefunctions typically have infinite average kinetic energy due to discontinuities of the wavefunctions and their derivative at the boundary, which is clearly an unrealistic scenario. Thus, the pointer states must have infinite support, and so their mutual overlap cannot be zero. It can be made sufficiently small, however, so that the state are at least quasi-orthogonal to each other, which suffices to reproduce the predictions of standard quantum mechanics to a degree that is only limited by the technological state of the art.

The nature of the measurement interaction is such that during a short measurement period $T_{M}$, the system of interest $S$ is coupled to the measurement device $M$ by a strong interaction $\hat{W}_{S M}$, so that the unperturbed Hamiltonian can be neglected,

$\hat{H}_{S}+\hat{H}_{M}+\hat{W}_{S M} \approx \hat{W}_{S M}$.

Before and after the measurement period, the interaction term is zero, so that the system of interest and the measurement device evolve independently from each other. The shortness of the measurement period $T_{M}$ can be more precisely defined by the requirement that the free evolution of the system of interest during a period of length $T_{M}$ can be neglected, that is

$e^{-i \hat{H}_{S} T_{M}} \approx \mathbb{1}$.

During a measurement of some observable $\hat{A}=\sum_{a} a \hat{\Pi}_{a}$, the system of interest becomes entangled with the measurement device, so that each pointer state $\eta_{a}$ becomes correlated with the projection of the wavefunction $\psi$ of the system of interest on the subspace corresponding to the eigenvalue " $a$ "; thus

$\psi \otimes \eta_{R} \rightarrow \sum_{a} \hat{\Pi}_{a} \psi \otimes \eta_{a}$,

where $\eta_{R}$ is the "ready" state of the measurement device.

As an example $[4,17,53]$, the interaction term may be given by

$\hat{W}_{S M}=-g\left(\hat{A} \otimes \hat{p}_{z}\right)$,

where $g$ is a sufficiently large coupling constant and $\hat{p}_{z}$ is the momentum operator conjugate to the configuration operator $\hat{z}$ of the measurement device. Because of (51) the state of the total system after measurement reads

$$
\begin{aligned}
e^{i g\left(\hat{A} \otimes \hat{p}_{z}\right) T_{M}}\left[\psi \otimes \eta_{R}\right] & =\sum_{a} \hat{\Pi}_{a} \psi \otimes e^{i g a T_{M} \hat{p}_{z}} \eta_{R} \\
& =\sum_{a} \psi_{a} \otimes \eta_{a},
\end{aligned}
$$

where the functions

$\eta_{a}(z)=\eta_{R}\left(z-g a T_{M}\right)$

each have an effective support given by

$Z_{a}=\left\{z \mid z-g a T_{M} \in Z\right\}$,

where $Z$ is an effective support of the "ready" state $\eta_{R}$. Depending on the measurement duration $T_{M}$ and on the separation of the eigenvalues $a$, the coupling constant $g$ must be chosen large enough, so that condition (50) is met.

Directly after measurement the wavefunction $\Psi^{\prime}$ of the total system is a sum of branches,

$\Psi^{\prime}=\sum_{a} \Psi_{a}^{\prime}$, 
with each branch

$\Psi_{a}^{\prime}=\hat{\Pi}_{a} \psi \otimes \eta_{a}$

representing a different measurement outcome corresponding to the eigenvalue " $a$ " of the observable $\hat{A}$. Because of (50) the branches are approximately orthogonal, so we have

$\left|\Psi^{\prime}\right|^{2} \approx \sum_{a}\left|\Psi_{a}^{\prime}\right|^{2}$

The measurement has the outcome " $a$ " in world $w$ when the configuration of that world lies within the region $Q_{a}=\mathcal{Q}_{S} \times Z_{a}$, which is a formal way of stating that in world $w$ the pointer shows a value corresponding to the outcome " $a$ ". So far the objective description. In order to see what happens in a particular world, we have to go to the subjective description from the perspective of a particular observer Joe. Denote the world-instance of observer Joe in world $\bar{w}$ by $\overline{J o e} \equiv J o e @ \bar{w}$, and denote by $\bar{q}$ the configuration of world $\bar{w}$ right after the measurement. By construction, Joe will read off the measurement result " $a$ " if and only if he finds the pointer in one of the configurations contained in $Z_{a}$, which means for the total system that the configuration $\bar{q}$ of Joe's world $\bar{w}$ must be contained in the region $Q_{a}=\mathcal{Q}_{S} \times Z_{a}$. The probability for this to happen reads according to (15)

$$
\begin{aligned}
P\left(\bar{q} \in \mathcal{Q}_{a}\right) & =\frac{\mu\left(Q_{a}\right)}{\mu(\mathcal{Q})}=\frac{\int_{Q_{a}} \mathrm{~d} q\left|\Psi^{\prime}(q)\right|^{2}}{\int \mathrm{d} q\left|\Psi^{\prime}(q)\right|^{2}} \\
& \approx \frac{\int_{\mathcal{Q}_{\mathcal{S}}} \mathrm{d} x \int_{Z_{a}} \mathrm{~d} z \sum_{a^{\prime}}\left|\hat{\Pi}_{a^{\prime}} \psi(x)\right|^{2}\left|\eta_{a^{\prime}}(z)\right|^{2}}{\|\Psi\|^{2}} \\
& \approx \frac{\int_{\mathcal{Q}_{S}} \mathrm{~d} x\left|\hat{\Pi}_{a} \psi(x)\right|^{2}}{\|\Psi\|^{2}} \\
& =\frac{\left\|\hat{\Pi}_{a} \Psi\right\|^{2}}{\|\Psi\|^{2}},
\end{aligned}
$$

where we have used (61) and (49). So the probability that Joe obtains the measurement result " $a$ " approximately coincides with the probability given by the Born rule (4). The degree of the approximation depends on the spatial separation of the pointer wave packets after measurement, which in turn depends on the strength and duration of the measurement interaction.

Let us go further and derive the "collapse of the wavefunction", which here becomes a merely subjective collapse experienced in each world separately and differently. Let $t_{M}$ be the time immediately after the measurement is finished, so that the post-measurement wavefunction is given by $\Psi_{t_{M}}$. For $t>t_{M}$ the wavefunction will evolve according to

$\Psi_{t}=\hat{U}\left(t-t_{M}\right) \Psi_{t_{M}}$.

With $\bar{\gamma}$ being the trajectory of the universe in world $w$, the post-measurement configuration of Joe's world reads $\bar{q}=\bar{\gamma}_{t_{M}}$. From the moment right after the measurement, the trajectory of Joe's world will evolve according to (33), with the point $\bar{q} \in Q_{a}$ fixing which trajectory is his one. Since the Bohm equation (20) is hyperbolic, the further course of Joe's world for $t>t_{M}$ depends on the new "initial" configuration $\bar{q}$. By construction $\bar{q}$ is somewhere in $Q_{a}$ at time $t_{M}$, and for any $\bar{q} \equiv(\bar{x}, \bar{z})$ in $Q_{a}$, we have $\bar{x} \in \mathcal{Q}_{S}$ and $\bar{z} \in Q_{a}$, and so the wavefunction of $\Psi_{t_{M}}$ evaluated at $\bar{q}$ reads

$$
\begin{aligned}
\Psi_{t_{M}}(\bar{q}) & =\sum_{a^{\prime}} \hat{\Pi}_{a^{\prime}} \psi(\bar{x}) \eta_{a^{\prime}}(\bar{z}) \\
& \approx \hat{\Pi}_{a} \psi(\bar{x}) \eta_{a}(\bar{z}) \\
& =\left(\hat{\Pi}_{a} \otimes \mathbb{1}\right) \Psi_{t_{M}}(\bar{q}),
\end{aligned}
$$

where we have used that $\eta_{a^{\prime}}(\bar{z}) \approx 0$ for $a^{\prime} \neq a$. Thus, from the moment right after the measurement, the wavefunction that governs the future fate of $\overline{\mathrm{Joe}}$ 's world becomes subjectively equal to the collapsed wavefunction $\bar{\Psi}_{a}:=$ 
$\left(\hat{\Pi}_{a} \otimes \mathbb{1}\right) \Psi_{t_{M}}$, although the wavefunction is objectively uncollapsed. From now on, since the Schrödinger equation is linear, the future fate of $\overline{J o e}$ 's world is subjectively governed by the time-evolved collapsed wavefunction

$\bar{\Psi}_{a, t}=\hat{U}\left(t-t_{M}\right) \bar{\Psi}_{a}$,

where $t>t_{M}$. In contrast to Everettian mechanics, there is no splitting of worlds. Before and after the measurement the total amount of worlds is the same and given by $\mu_{t}(\mathcal{Q})$, no worlds are being created or destroyed, split or combined. What happens is that due to the measurement process, the configuration space is partitioned into smaller volumes that contain those worlds where the individual measurement outcomes occur. As the theory is deterministic at the level of individual worlds, which follows from the unique solvability of the Bohr equation (33), the measurement result obtained in each individual world is determined from the very beginning (see [48] for a similar view on determinism in quantum mechanics, including a critical review on the ideas proposed in [5]). It only appears to be random to the individual observer who spends their lifetime in a particular trajectory without knowing which one. The conundrum of the splitting of persons that occurs in Everett's theory does not show up (see [38], however, for an intriguing analysis making the splitting of persons less bizarre).

Concluding, the here-proposed theory explains (1) the subjective occurrence of probabilities, (2) their quantitative value as given by the Born rule, and (3) the apparently random "collapse of the wavefunction" caused by the measurement process and by the subjective experience of individual observers, while remaining an objectively deterministic theory.

\section{Discussion}

Let me discuss certain aspects of my approach, in particular with respect to theories, ideas and concepts found in the literature, which are more or less close to the ideas put forward here. The following topics are not ordered by their importance or by the similarity of the discussed concepts to my approach, but rather the ordering tries to follow a thematic thread.

\subsection{Bohmian mechanics}

Although the here-presented theory may be considered a variant of Bohmian mechanics (see [8, 14,20,32,34], for excellent reviews), there are conceptual differences between the two theories, in particular with regard to certain critical issues.

The first issue is related to the empirically undeniable statistical character of the measurement results. Just like Everettian mechanics, Bohmian mechanics is a deterministic theory, and there seems to be prima facie no reason why the particles occupy one branch of the post-measurement state rather than another, with a probability whose value is precisely given by Born's rule (4). The proponents of Bohmian mechanics argue that the probability that appears in Born's rule is an epistemic quantity related to the ignorance of the observer concerning the initial particle configuration. In analogy to classical statistical mechanics, so their argument, one must then introduce a probability density $\rho$ on configuration space that captures the ignorance about the actual configuration which is a result of the ignorance about the initial configuration. The predictions of Bohmian mechanics are indistinguishable from those of conventional quantum mechanics, exactly if

$\rho_{t}=\left|\Psi_{t}\right|^{2}$

for some arbitrary initial time $t$. The dynamical laws then guarantee that (71) holds for all times $t$, a feature that is denoted as equivariance. So, Born's rule is replaced by relation (71) which, in lack of a derivation, has the status of a hypothesis, and it is called the quantum equilibrium hypothesis. From there, with the help of the dynamical laws, the Born rule can be derived, so it no longer exists as an additional postulate. There are attempts to derive the quantum equilibrium hypothesis at least in an approximative manner. Valentini has shown that any arbitrary initial probability density on the configuration space becomes eventually indistinguishable from $\left|\Psi_{t}\right|^{2}$ at a coarsegrained scale [49]. His theorem is partly analogous to Boltzman's famous H-theorem, which motivates Valentini to 
name his theorem the subquantum H-theorem. Dürr et al. propose to consider the quantum equilibrium as a feature of typical initial configurations [14]. However, I do not consider these justifications of the quantum equilibrium hypothesis satisfactory, for reasons that go beyond the scope of this paper and have to be outlined separately. In the world continuum theory, there is no quantum equilibrium hypothesis, and all probabilities emerge as epistemic probabilities caused by the ignorance of the observer about which world it is that they live in.

The second issue with Bohmian mechanics may at first sight appear rather harmless, but on a closer look it develops considerable destructive power: the issue of empty branches. These are the components of the postmeasurement state that do not guide any particles because they do not have the actual configuration in their support. At first sight, the empty branches do not appear problematic but on the contrary very helpful as they enable the theory to explain unique outcomes of measurements. Also, they seem to explain why there is an effective "collapse of the wavefunction", like in standard quantum mechanics. On a closer view, though, one must admit that these empty branches do not actually disappear. As in Bohmian mechanics the wavefunction is taken to describe a really existing field, all their branches really exist and will evolve forever by the Schrödinger dynamics, no matter how many of them will become empty in the course of the evolution. This circumstance has led David Deutsch to famously phrase that "pilot-wave theories are parallel-universes theories in a state of chronic denial" ([9]; this is a comment on [28]; for a follow-up discussion see [6,51]). Every branch of the global wavefunction describes a complete world which is, according to Bohm's ontology, only a potential world that would be actual if only it were filled with particles. Exactly one branch at a time is occupied by particles, thereby representing the actual world, while all other branches, though really existing as part of a really existing wavefunction, are empty and thus describe some sort of "zombie worlds" with potential planets, oceans, trees, cities, cars, and potential people who would talk like us and behave like us, but who do not actually exist. The empty branches of the wavefunction are still real, because the entire wavefunction is considered to be real, but they have no further influence on the particles. So, is there any convincing justification to consider empty branches still as real, beyond mere stipulation? Why is the effective collapse of the wavefunction not a real collapse? If a many-worlds theory may be accused of ontological extravagance, then Bohmian mechanics may be accused of ontological wastefulness. Because, on top of the ontology of the wavefunction with all its branches comes the additional ontology of particles, whose actual configuration degrades the reality of most of the branches of the wavefunction into mere potentiality. Yet, the actual configuration is never needed for the calculation of the statistical predictions in experimental reality, for these can be obtained by mere wavefunction algebra. In the world continuum theory, in contrast, there is no such thing as the actual configuration. All configurations in the support of the wavefunction are equally real, and the objective description of the universe does not need a specification of one of these configurations being the actual one. Probability comes into play not as the ignorance of observers about which configuration is the actual one, but rather as their ignorance about which configuration is the configuration of their world, in a sense that is precisely specified by the here-provided logical framework.

The third issue with Bohmian mechanics is the separate existence of wavefunction and particles and the strange way that these entities interact with each other. While the wavefunction acts upon the particles, the particles do not act upon the wavefunction. So actually, there is no interaction between the wavefunction and the particles; the relation is asymmetric. However, although the particles never act back on the wavefunction, it is always the particles that define the unique outcome of measurements; it is the particles that define which branch of the wavefunction is the relevant one while the other branches become empty and can be neglected. So, although the particles have the last word, they are yet so powerless that they cannot even act upon the wavefunction. In the world continuum theory, in contrast, there is no separate existence of wavefunction and particles, and no bizarre one-way "inter"-action between these entities. There is only one unified physically existing entity, the world continuum, and the wavefunction is just an abstract mathematical construct that contains all information needed to determine the form of the world continuum. Moreover, in the world continuum theory, particles are not point-like entities like in Bohmian mechanics, but rather all particles together constitute a continuous substance, the world continuum, and it is just the time-world instances of the world continuum that appear as discrete point-like particles. In the world continuum theory, thus, the dualism between the continuum and the discrete, between wave and particle, is resolved in a unique fashion. Concluding, although being formally equal to Bohmian mechan- 
ics in many aspects, the world continuum theory draws an entirely different picture of the physically existing universe.

\subsection{Mind and world}

I will not say anything about the relation between mind and world that goes beyond the absolute minimum. "If there exist many worlds", the opponent might ask, "then why is it that we experience only one of them?" The simplest answer is: "For reasons analog to those that make us experience only one time point as the present time". A more elaborate answer requires one to agree that perception is a conscious process and that conscious processes supervene on physical processes. That is to say, a conscious process is taken to be uniquely determined by physical processes. If all physical processes are given then all conscious processes are also given. As all physical processes are determined by the movement of the particles in the universe, a conscious process is eventually determined by the temporal evolution of the configuration of the universe, which is given in each world by the universal trajectory in that world. In some world $w_{1}$ the universal trajectory is $\gamma_{1}$, in some other world $w_{2}$ the universal trajectory is $\gamma_{2}$. Hence, in some world $w_{1}$ at time $t$, Joe is in a certain mental state $M_{1}$ uniquely determined by the trajectory point $\gamma_{1, t}$, and in some other world $w_{2}$ at the same time $t$, Joe is in some other mental state $M_{2}$ uniquely determined by the trajectory point $\gamma_{2, t}$. In no world, however, Joe is in the mental states $M_{1}$ and $M_{2}$ together at the same time. In the same manner that traffic lights cannot be green and red at the same time in the same world, Joe cannot be in the mental states $M_{1}$ and $M_{2}$ at the same time in the same world. Roughly speaking, Joe cannot be experiencing more than one world, because his experience is part of the world, so in different worlds Joe has different experiences. Say, at time $t$ Joe is in one world $w_{1}$ experiencing green traffic lights, and in another world $w_{2}$ he is experiencing red traffic lights. Although there is no logical contradiction in Joe experiencing both green and red traffic lights at the same time, because he does so in different worlds, it may seem less confusing to talk about different worldinstances of Joe experiencing the traffic lights either as red or as green at time $t$. So rather than speaking of Joe being in a mental state $M_{1}$ at time $t$ and in world $w_{1}$, while being in another mental state $M_{2}$ at the same time $t$ in another world $w_{2}$, one may equivalently speak of one world-instance of Joe, namely Joe $1 \equiv J_{0 e} @\left(w_{1}\right)$ being in one mental state $M_{1}$ at time $t$, and another world-instance of Joe, namely Joe $2 \equiv \mathrm{Joe}_{(}\left(w_{2}\right)$ being in another mental state $M_{2}$ at time $t$. There is no instance of Joe that is in both mental states $M_{1}$ and $M_{2}$ at time $t$.

It is difficult to decide whether and to what extent there might be causal connections between worlds, like there are causal connections from past world-instances to future world-instances. If there were such inter-world causation, there would be the possibility of imprints in an observer's brain in a specific world caused not only by events occurring in past instances of that world, but also by events in parallel worlds. This would entail the possibility of "counterfactual sensing" analogue to having a memory. The many-interacting-worlds (MIW) approach by [21], which bears many similarities to my approach, seems to support the possibility of inter-world causation. However, as I will substantiate further on, I would rather not regard the relation between worlds as interaction but as interference, which may have consequences on the possibility of counterfactual sensing. In any case a closer analysis, which also entails taking into account different concepts of causation, is needed though clearly outside the scope of this paper.

\subsection{Tipler's approach}

There is a very interesting formulation of quantum mechanics by Tipler [43] which seems to be similar in spirit to the ideas proposed here. The author explicitly writes [43, page 1]:

The key idea of this paper is that the square of the wave function measures, not a probability density, but a density of universes in the multiverse.

Unfortunately, though, Tipler deviates from his initial conception when, for example, he later writes [43, page 4]: 
In the case of spin up and spin down, there are only two possible universes, and so the general rule for densities requires us to have the squares of the coefficients of the two spin states be the total number of effectively distinguishable—in this case obviously distinguishable—states.

Such statement is hard to understand. If the number of universes (or "worlds", as the author also calls them elsewhere in the paper) is two, then what does it mean to "have the squares of the coefficients of the two spin states be the total number of effectively distinguishable [...] states"? The word "state" seems to refer to a universe, or world, and within the same sentence also to something else. How many "states" or "universes" are there, in that situation, two or infinitely many? Such ambiguity and vagueness about the ontological meaning of the terms "states", "branches", "worlds", "universes", is somewhat idiosyncratic for Everett-type theories. In the theory proposed here, in contrast, worlds correspond to well-defined trajectories in configuration space and hence their total number is always uncountably infinite, and spin states are just components of the wavefunction and not labels for, or representatives of, worlds. It seems that after all Tipler sticks to the Everettian ontology of branches rather than to a continuous multiplicity of worlds, in contrast to what the author's initial statement seems to suggest. Other strong indicators for that conclusion are that in Tipler's analysis the universes still split, or "differentiate", as the author also calls it, and that he explicitly writes "the sums in (15) [...] are in 1 to 1 correspondence with real universes", where the referenced formula involves a decomposition of the wavefunction into spin states. Another fundamental difference to the here-proposed theory concerns the justification of probabilities. Tipler writes [43, page 1-2]:

The probabilities arise because of the existence of the analogues of the experimenters in the multiverse, or more precisely, because before the measurements are carried out, the analogues are 'indistinguishable' in the quantum mechanical sense. Indistinguishability of the analogues of a single human observer means that the standard group transformation argument used in Bayesian theory to assign probabilities can be applied. I show that the group transformation argument yields probabilities in the Bayesian sense and that in the limit of an infinite number of measurements, the relative frequencies must approach these probabilities.

Different from this rather sophisticated justification, the probabilities in the here-proposed theory derive from a straightforward generalization of the Laplacian rule to a continuum of possibilities. I conclude that Tipler's approach is conceptually different from mine.

\subsection{The MIW approach}

Bohmian mechanics can be formulated, so that the Bohr equation (20) obtains a Newtonian form

$m_{n} \ddot{\gamma}_{n}=-\nabla_{n}\left(V_{t}+Q_{t}\right)$,

for $n=1, \ldots, N$, involving the so-called quantum potential

$$
Q_{t}=\sum_{n=1}^{N}-\frac{\hbar}{2 m_{n}} \frac{\nabla_{n}^{2} \sqrt{\rho_{t}}}{\sqrt{\rho_{t}}},
$$

and with the initial velocity of the particles being restricted by

$\dot{\gamma}_{n, 0}=\frac{\dot{j}_{n, 0}\left(\gamma_{0}\right)}{\rho_{0}\left(\gamma_{0}\right)}$.

In this formulation, which was presented by Bohm himself [3,4], it becomes explicit how the motion of the particles is affected not only by classical forces but also by non-classical forces generated by the quantum potential, which vanishes in the classical limit $\hbar \rightarrow 0$. Since the quantum potential is a function of the density $\rho_{t}=\left|\Psi_{t}\right|^{2}$, Bohm interpreted the non-classical force as the force that the wavefunction exerts on the particles. 
In a very interesting approach, Hall et al. [21] propose to replace the density $\rho_{t}$ in (73) with the empirical density $\rho_{t}^{K}$ of a discrete number of trajectories, identified as worlds, so that

$\rho_{t}^{K}(q)=\frac{1}{K} \sum_{k=1}^{K} \delta\left(q-\gamma_{k, t}\right)$,

where $K$ is the number of worlds. That way, the one-way interaction between the wavefunction and the particles becomes a two-way interaction between worlds, in that the evolution of each world configuration $\gamma_{k, t}$ is directly determined by all other world configurations. If the worlds are distributed by a probability density equal to $\left|\Psi_{t}\right|^{2}$, then for $K \rightarrow \infty$ the world density $\rho_{t}^{K}$ approaches (in a distributional sense) the standard density, $\rho_{t}^{K} \rightarrow\left|\Psi_{t}\right|^{2}$; hence the theory would reproduce the predictions of standard quantum mechanics in the limit of infinitely many worlds.

I find this proposal very fascinating, as it shows that quantum effects can be explained by the interaction between physically existing worlds. I have only three criticisms. One is that I would prefer to call the relation between worlds not interaction but rather interference, because the difference between interaction and interference seems to me a categorical one. Interaction is a relation between systems, while interference is a relation between worlds, that is, between trajectories of one and the same system. For example, an electron in a double-slit experiment interacts with the walls of the double-slit and with the detection screen behind the slits. On the other hand, a trajectory of the electron passing through one slit interferes with the trajectories of the same electron passing through the other slit. If one of the slits is closed, then the trajectories of the electron cannot pass the region covered by the closed slit, and all trajectories go through the open slit, interfering with each other and producing a typical single-split diffraction pattern on the screen when the experiment is repeated many times. When the second slit is opened, the trajectories of the particle may pass both slits, and behind the double-slit they interfere with each other, producing a typical double-slit interference pattern on the screen. In each world there is only one trajectory passing through only one of the slits, but since the course of that trajectory is influenced by other trajectories, each belonging to a separate world, one is led to conclude that the typical wave phenomena of particles can be interpreted as the influence that individual worlds exert on one another. This special kind of mutual influence between worlds is different in nature from ordinary interactions between systems and, therefore, so my suggestion, should be distinctively identified as interference.

My second criticism of the approach of [21] is, of course, that they assume a finite number of worlds. For one part, if the predictions of standard quantum mechanics are reproduced in the limit of infinitely many worlds, then why not taking infinitely many worlds? The here-proposed theory already involves right from the start an infinite number of worlds while being mathematically much simpler than the proposal by Hall et al. The world continuum theory may be regarded as the limiting theory obtained from the many-interacting-worlds (MIW) theory of Hall et al. by taking the number of worlds to infinity.

My third criticism is that the MIW relies, like Bohmian mechanics, on a probability assumption. Only if it is assumed that the finitely many worlds are at $t=0$ spatially distributed by $\left|\Psi_{0}\right|^{2}$, in the sense of a probability distribution, the predictions of standard quantum mechanics are approximately reproduced. Thus, it is impossible to conceptually derive probability from the MIW. Nonetheless, the authors claim that the worlds are equally weighted, so that a probability argument analog to that of Laplace would be applicable [21, p 041013]:

It is a remarkable feature of the MIW approach that only a simple equal weighting of worlds, reflecting ignorance of which world an observer occupies, appears to be sufficient to reconstruct quantum statistics in a suitable limit.

At a closer look, there are two types of probability brought into play here. One is the probability $p_{k}$ that world $k$ is the world of the observer, and the other is the probability density $\rho_{k, 0}(q)$ to find at time $t=0$ the configuration of world $k$ at $q$. Due to the observer's complete ignorance which world it is that they live in, the probability $p_{k}$ is equally weighted, so $p_{k}=1 / K$. In the same vein, the probability density $\rho_{k, 0}$ is assumed to be equal for all worlds, so $\rho_{k, 0}=\left|\Psi_{0}\right|^{2}$. Importantly, while each world is given the same probabilistic weight with respect to its bare identity, which can be justified by an ignorance assumption, its possible configurations are not given the 
same probabilistic weight. Some configurations are more probable than others, and there is no simple ignorance argument for such a non-trivial probability assumption. So my criticism is that the authors conceal a non-trivial and not justified probability assumption concerning the configuration of the world of the observer by a trivial and justified probability assumption concerning the bare identity of the world of the observer. They do not address the following question: why are some configurations more probable than others, and where does that non-uniform probability come from? As a side remark: it does not help in this respect to formally parametrize the worlds by some parameter $C$, so that $C$ becomes uniformly distributed as is done by Schiff and Porier in their approach, which is conceptually very close to mine [39]. For, the explanatory burden is now shifted towards answering the following question: where does that parameterization come from? It appears ad hoc to introduce a specific parameterization of worlds only to get the probability distribution over the new parameter $C$ uniform, as long as there is no physical justification for doing so. Why should the observer apply the Laplacian rule of indifference towards some fictitious parameter $C$, when it is really and only the positions of particles, that is, the configuration of the world, which he can (in principle) measure? The parameter $C$ is a merely theoretic, not an empirical, quantity that depends on the probability distribution over the possible world configurations, so it would be circular to use it for a derivation of that very probability distribution.

As I have argued in Sect. 3.3, there is an epistemological explanation for the occurrence and the numerical values of quantum mechanical probabilities when interpreting $\left|\Psi_{t}\right|^{2}$ not as a probability density but rather as the substantial density of a physically existing continuum of worlds. Probability can then be conceptually derived, in a manner analogous to the classical derivation by Laplace, from both the physical facts (the empirically given distribution of the world continuum) and the observer's indifference towards which one out of the continuously many worlds is actually the world of the observer. The configuration of the world of the observer is more likely to be found near the location $q$ than near another location $q^{\prime}$ exactly if $\rho(q)>\rho\left(q^{\prime}\right)$, since at $q^{\prime}$ the worlds are more densely packed than at $q^{\prime}$. As a discrete analogue: It is more probable to find a particular needle out of many needles in a haystack if you look at those places where there are more needles. You do not even have to know the distribution of needles in the stack. The probability to find your needle is, as a matter of physical fact, bigger when you (accidentally) look at places where there are more needles. The probability itself is epistemic, but it derives from ontological facts. Now, the interpretation of $\left|\Psi_{t}\right|^{2}$ as a substantial density of worlds is impossible when considering an only finite number of worlds. As such, the substantial density is an empirical distribution, and if there were only finitely many worlds, the empirical distribution had to be a finite sum of delta functions, which does not apply for $\left|\Psi_{t}\right|^{2}$.

\subsection{Newtonian QM}

Sebens [40] considers a large but finite number of worlds in his proposal of a Newtonian QM. He completely removes the wavefunction from the theory, leaving only the density $\rho=\rho_{t}(q)$ and the velocity field $v=v_{t}(q)$, which together yield the flow $j=\rho v$. With the wavefunction having been removed, the Newtonian equation (72) for the movement of the particles cannot, as in Bohmian mechanics, be interpreted as the action of a classical potential and a quantum field on each particle. Instead, the equation seems to describe the action of a classical potential and a classical continuous fluid described by the density $\rho$ on each particle. However, according to Sebens' construction of the theory, there is no continuous fluid but just a finite number of worlds, and the density $\rho$ is just an approximation obtained by averaging over a large enough number of worlds. Hence, as Sebens points out, (72) cannot be considered a fundamental equation, so that empirically testable deviations may occur. Furthermore, as the wavefunction is no longer there to put restrictions on the density and the velocity field, there are combinations of the latter two that do not correspond to any underlying wavefunction and, therefore, do not represent empirically adequate scenarios. To restore the empirical adequacy of the theory, Sebens introduces an additional postulate, the "quantization condition", which holds that

$$
\oint\left\{\sum_{k}\left[m_{k} \boldsymbol{v}_{k} \cdot \mathrm{d} \boldsymbol{l}_{k}\right]\right\}=n h .
$$


Sebens admits that he has no satisfying justification for the additional postulate other than that it would naturally follow if one presumes the existence of an underlying wavefunction. However, with the wavefunction existing in addition to a large number of worlds, the theory blows up to an ontologically extravagant theory, Prodigal QM, which does not offer interpretational advantages against standard Bohmian mechanics. Therefore, he introduces the wavefunction again, though holding that it does not represent a physically existing entity itself, but rather "a convenient way of summarizing information about the positions and velocities of particles in the various worlds" [40, p. 9].

I find Sebens' approach and his interpretational analysis particularly useful in that it clearly shows at which points problems occur that have to be addressed by additional conceptual work. My own conclusions are (1) to accept that there is a continuous infinity of worlds corresponding to Bohmian trajectories (here I differ from Sebens), and (2) to interpret the wavefunction not as a physically existing field in addition to particles and worlds, but rather as a generating function for, and, therefore, as a mathematical representation of, a physically existing entity that already entails particles and worlds (which is more or less what Sebens also concludes).

\subsection{Poirier and Schiff's approach}

Perhaps the approach closest to my proposal is Poirier and Schiff's reformulation of Bohmian mechanics [35,39]. In a sense, their formulation and mine are two formulations of the same theory, only emphasizing different aspects of it. While my formulation puts a focus on the interpretation of the density function $\rho$, as well as on the ontological and epistemological aspects of the theory, Poirier and Schiff's formulation explores formal aspects and their physical relevance. Their main result is that one may completely get rid of the wavefunction, leaving only the trajectory function $\xi=\xi_{t}(q)$ and the initial density function $\rho_{0}=\rho_{0}(q)$ as a complete representation of the history of a closed system. ${ }^{3}$ At first, Poirier [35] showed that the entire dynamics of a 1D system is captured by a partial differential equation (PDE) for the trajectory function $\xi=\xi_{t}(q)$, involving temporal derivatives $\dot{\xi}, \ddot{\xi}$ as well as spatial derivatives $\xi^{\prime}, \xi^{\prime \prime}, \xi^{\prime \prime \prime}$. He then showed that the system dynamics can be expressed in terms of a minimal-action principle on a generalized Lagrangian density of the form

$\mathcal{L}\left[q, \xi, \dot{\xi}, \xi^{\prime}, \xi^{\prime \prime}, \xi^{\prime \prime \prime}\right]=\rho_{0}(q) L\left[q, \xi, \dot{\xi}, \xi^{\prime}, \xi^{\prime \prime}, \xi^{\prime \prime \prime}\right]$

with the Lagrangian

$L\left[q, \xi, \dot{\xi}, \xi^{\prime}, \xi^{\prime \prime}, \xi^{\prime \prime \prime}\right]=\frac{1}{2} m \dot{\xi}^{2}-V[\xi]-Q\left[q, \xi^{\prime}, \xi^{\prime \prime}, \xi^{\prime \prime \prime}\right]$

and a rather complicated quantum potential $Q$. Schiff and Poirier [39] generalized the theory to the many- $D$ case, and they also considerably simplified $Q$ and the PDE by re-parameterizing the trajectory function $\xi_{t}(q)$ to $\xi_{t}(C)$ with the parameter $C$ being chosen, so that it uniformizes the probability density $\rho_{0}(q)$ to $\rho_{C}(C) \equiv 1$, where $\rho_{C}(C) \mathrm{d} C=\rho_{0}(q) \mathrm{d} q$. The relevance of their work to the understanding of quantum mechanics is profound. For one part it shows that a complex-valued wavefunction need not be considered an indispensable ingredient to quantum theory. For another part, and alongside with $[21,40]$, the work of Poirier and Schiff shows that quantum phenomena can be regarded as being caused by the interaction (or, as I would prefer to call it, by the interference) of a multitude of equally existing trajectories. For, if one were to reject the idea of equally existing trajectories, then one would have to justify how and in what sense a lonely existing trajectory can possibly interact (or interfere) with other, non-existing, trajectories.

Although in my approach the wavefunction is still there, it is degraded to a mere "generating function" for the physically existing trajectories, with no reality on its own. In this respect, both of our approaches go beyond what Bohm and his adherents had, respectively, have, in mind. The trajectories are the same in all three theories, and they form a continuum, so this is common ground, too.

\footnotetext{
${ }^{3}$ Here and in the following I adapt their notation to mine for the sake of easier comparison.
} 
However, what Porier and Schiff did not fully work out, and what is more closely addressed in my approach, is the physical meaning of the collection of trajectories and of the density function. Porier and Schiff refer to the collection of trajectories as an ensemble, which, however, remains a rather ambiguous reference. In statistical theories, an ensemble is a hypothetical, infinite collection of independent realizations of a random variable, and the probability distribution associated with the outcomes of the variable governs the relative frequencies of individual realizations within the ensemble. A statistical ensemble is in any case countably infinite, even for a finite number of possible values of the random variable under consideration. For example, the ensemble associated with a fair coin would be an infinite sequence of heads and tails, with the relative frequency of heads within a partial sequence converging towards one half as the length of the partial sequence goes to infinity. This is certainly not the concept of ensemble that Poirier and Schiff intend to invoke. Rather, their "ensemble" of trajectories is the uncountably infinite collection of all possible trajectories, which are parameterized solutions of one and the same differential equation. In terms of a statistical theory, their "ensemble" would rather represent the (uncountable) collection of all possible outcomes, not a (countable) collection of realizations, of a random trajectory. The density function $\rho$ would then represent the probability density on the collection of possible trajectories, with only one trajectory being realized. Poirier seems to sympathize with this view, which coincides with the standard view on Bohmian mechanics, when he writes [35, p. 14]:

Perhaps the most natural association between the present formulation and existing interpretations of quantum mechanics would be with the statistical or ensemble interpretation [cit.], whose most famous proponent was Einstein himself. Indeed, the trajectory density weighting function, $\rho_{0}\left(x_{0}\right)$, may be regarded as a classical statistical probability distribution [...].

At several places in the paper [35, pp. 4, 9, 10, 13, 14], Poirier calls $\rho$ a "trajectory density weighting", but he does not explicate what this actually means. At other places again [35, pp. 6, 8, 9, 11, 13, 14], the author stays with the standard notion of "probability". Also, there is no clear commitment as to how his approach relates to the existence of parallel worlds. On page 14 we read

If one presumes objective existence for a single trajectory only, then the remaining trajectories in the ensemble must be regarded as "virtual", in some sense. On the other hand, one might prefer to regard all trajectories in the quantum ensemble as equally valid and real. It is hard to imagine how this could be achieved, without positing that each trajectory inhabits a separate world. It must be emphasized, however, that this version of the many worlds interpretation would be very different from the standard form [cit.].

In their later paper Schiff and Poirier still leave open how to interpret their formal framework [39, p. 031102-4]:

Regarding interpretation, we draw no definitive conclusions here. However, it is clearly of great significance that the form of $Q$ can be expressed in terms of $x$ and its $C$ derivatives-implying the key idea that the interaction of nearby trajectories, rather than particles, is the source of all empirically observed quantum phenomena (suggesting a kind of "many worlds" theory, albeit one very different from [Everett's]).

Somewhere else again in the paper, the authors explicitly speak of $\rho_{0}\left(x_{0}\right)$ as of a "probability density" [39, p. 0311022 ]. Even if one is willing to read their approach in the spirit of a many-worlds theory, in such a way that all members of the collection of trajectories (their "ensemble" of trajectories) are taken to equally exist, it remains unclear how to interpret the density function $\rho$. If it were not a probability density, what kind of density could it possibly represent? Calling it a density weighting at times [35, pp. 5, 9, 10, 13, 14] does not really help as it still leaves open what kind of "weighting" is mediated by $\rho$ in their theory (if not a probability weighting).

I conclude that the approach by Poirier and Schiff bears great similarities to my proposal, but its physical interpretation remains too vague to be considered a complete equivalent. My personal view is that Poirier and Schiff's approach and mine complement each other in that those questions having been left open by either approach are addressed by the other and that they are essentially about the same theory. 
6.7 Why should the worlds form a continuum?

In a recent paper, Vaidman [48] discusses numerous formulations and interpretations of quantum mechanics, and in particular the idea put forward in [43] and in [5], of a continuum of worlds existing in parallel. Although Vaidman strongly argues for a multiplicity of worlds, he rejects the idea that there is a continuum of them. Rather, so he argues, there can only be a large but finite number of parallel worlds. He writes [48, p. 24]:

If in any region of the configuration space there is an infinity of worlds, we cannot say that it is smaller or larger than in another region. [...] Boström apparently adopts the concept of the measure of existence. His proposal for "volume of worlds" (16) includes also the "weight" of each world $\left|\Psi_{t}(q)\right|^{2}$. But then, I do not see how it can be considered as a density of worlds. As far as I can understand, for an infinite number of worlds there is no mathematical formalism which can provide a "density of worlds" picture.

In the same context, with regard to Albert and Loewer's many-minds approach [1], where the authors propose a continuum of minds together with a measure on the totality of minds, Vaidman writes: "There is no mathematical formalism which provides such a measure". In line with Vaidman, Hall et al. [21] and Sebens [40] propose a large but finite set of worlds, which they identify with Bohmian trajectories. Although they admit that only in the limit of a continuum of worlds the predictions of standard quantum mechanics are exactly reproduced, they insist on a finite set. Sebens [40, p. 14] writes:

The meaning of $\rho$ becomes unclear if we move to a continuous infinity of worlds since we can no longer understand $\rho$ as yielding the proportion of all worlds in a given volume of configuration space upon integration over that volume. There would be infinite worlds in any finite volume (where $\rho \neq 0$ ) and infinite total worlds. If $\rho$ doesn't give the proportion of worlds in a region, it is unclear why epistemic agents should apportion credences as recommended in the previous section. So, the continuous variant, if sense can be made of it, faces the quantitative probability problem.

I do not share these worries. The mathematical formalism that I and certainly also the other cited authors tacitly refer to when considering a continuum of worlds (or minds) endowed with a measure, is measure theory. Briefly, a measure is a function $\mu$ on a collection $\Sigma$ of subsets of a set $\mathcal{X}$, which maps each subset $X \in \Sigma$ to a non-negative number $\mu(X)$ called the measure of $X$. The collection $\Sigma$ is called a $\sigma$-algebra of the set $\mathcal{X}$, and its elements are called $\mu$-measurable subsets of $\mathcal{X}$. Both the $\sigma$-algebra and the measure fulfill certain requirements. The cardinality of $\mathcal{X}$ does not matter; it might be finite, countable, continuous, or of even higher cardinality. Measure theory forms the basis of integral theory, for the Lebesgue integral $\lambda(X)=\int_{X} \mathrm{~d} x$ can be regarded as a special measure on the vector space $\mathcal{X}=\mathbb{R}^{N}$. Any measure on a Lebesgue-measurable space can be associated with a density $\rho$, such that $\mu(X)=\int_{X} \mathrm{~d} x \rho(x)$, with the Lebesgue measure itself having the trivial density $\rho \equiv 1$. As has already been discussed at some length, my proposed interpretation of a regular, i.e. non-singular, density in physical space is that of a distribution of a continuous substance (a fluid, say) across the space $\mathcal{X}$, so that $\mu(X)$ yields the amount of substance contained within a given region $X \subset \mathcal{X}$. In regions where the integrated density is higher, there is more substance than in other regions where the integrated density is lower. According to my proposal, the collection of all trajectories that solve the Bohm equation (20) constitutes a continuous substance distributed in configuration space, with each trajectory uniquely corresponding to an individual world. So $\mu_{t}(X)$ measures the amount of worlds whose configuration is contained at time $t$ within the region $X$.

If the density within a certain region $X$ is smaller than unity, one might be tempted to assume that in $X$ there must be holes, that is, places where there are no worlds. However, this is not necessarily the case. The worlds form a continuum, and a continuum does not have to have holes for its density to be decreased. As a simple toy model consider a set $\mathcal{X}=[0,1]$ of "worlds" being embedded into a set $\mathcal{Y}=[0,1]$ of "configurations" by the function $f(x)=x^{2}$. The function $f$ is a bijection here, so the set $\mathcal{Y}$ is entirely filled with points from $\mathcal{X}$ without any holes. Set theoretically, there are exactly as many points in $\mathcal{X}$ as in $\mathcal{Y}$, namely $\mathfrak{c}=2^{\aleph_{0}}$. Still, the density of points taken from $\mathcal{X}$ and embedded into $\mathcal{Y}$ is not uniform; there are "more" points (in the sense of a higher measure) from $\mathcal{X}$ contained in the lower end of $\mathcal{Y}$ than contained in the upper end. If $v$ is a measure on $\mathcal{X}$ then the measure $\mu$ on $\mathcal{Y}$ 
derived from $v$ is given by

$\mu(Y)=v(\{x \in \mathcal{X} \mid f(x) \in Y\})$.

So if we take the worlds in $\mathcal{X}$ to be uniformly distributed according to $v(X)=\int_{X} \mathrm{~d} x$, then we can explicitly calculate the measure $\mu$ on the configurations in $\mathcal{Y}$ as

$$
\begin{aligned}
\mu(Y) & =\int_{[0,1]} \mathrm{d} x \int_{Y} \mathrm{~d} y \delta(y-f(x)) \\
& =\int_{Y} \mathrm{~d} y \frac{1}{2 \sqrt{y}},
\end{aligned}
$$

and so the configurations of the worlds in $\mathcal{X}$ are distributed within $\mathcal{Y}$ according to the non-uniform density $\rho_{Y}(y)=$ $\frac{1}{2 \sqrt{y}}$. Then, the subjective probability that our actual world has a configuration within a certain region $Y \subset \mathcal{Y}$ is given by the ratio of the amount of worlds whose configuration lies within $Y$ divided by the amount of all worlds, so $P(Y)=\mu(Y) / \mu(\mathcal{Y})$. The probability that a particular world (for example our world) has a configuration within the interval $[0, a]$ for $0 \leq a \leq 1$ is given by $P([0, a])=\sqrt{a}$. The toy model illustrates how the notion of a density of worlds whose configurations are distributed across configuration space makes sense also in the case of a continuum of worlds.

Besides these rather mathematical aspects, why should one consider the set of worlds to form a continuum in the first place? Within the framework of my proposal, the answer is quite simple: because there is a continuum of trajectories that solve the Bohm equation (20). If only finitely many worlds were allowed, which of these trajectories should be taken as real, and for what reason? Logically, there is an infinite number of possible paths that the configuration of the universe may take. Invoking a differential equation (the Bohm equation) selects the physically possible paths from the logically possible ones, and the so-selected paths derive from one and the same wavefunction $\Psi_{t}$ representing the state of the universe. If only $\Psi_{t}$, and nothing more, is considered to represent a complete description of the universe, then all paths deriving from $\Psi_{t}$ must be considered as real. Discarding all but a finite number of paths is not only unnecessary and ad hoc, but it requires an additional postulate. And, if one allows for only a finite number of worlds then why not directly postulating that there is just one world, as is done in Bohmian mechanics? Finitely-many-worlds theories based on the conception of worlds as trajectories in configuration space, are axiomatically more demanding than the world continuum theory, as they must specify, in addition to the wavefunction, the trajectories $\gamma_{1}, \ldots, \gamma_{K}$ of those worlds that are taken as real, so that the objective state of the universe at time $t$ is given by

$$
\left(\begin{array}{c}
\Psi_{t} \\
\gamma_{1, t}, \ldots, \gamma_{K, t}
\end{array}\right)
$$

for $K$ representing the number of objectively existing worlds, and with $\gamma_{k}=\left(\boldsymbol{\gamma}_{k, 1}, \ldots, \boldsymbol{\gamma}_{k, N}\right)$ being the trajectory of the $k$-th world, whose configuration at time $t$ is given by $\gamma_{k, t}$. In the case $K=1$ one obtains Bohmian mechanics. The here-proposed world continuum theory, in contrast, just needs the wavefunction $\Psi$ to mathematically represent a continuum of trajectories and hence worlds, which are the solutions of the Bohm equation (20). Therefore, in the world continuum theory, just as in Everettian mechanics, the complete history of the universe is specified by the wavefunction alone. In contrast to Everettian mechanics, however, there is no ambiguity concerning the identity of the worlds, as they are given by precisely defined trajectories. To explain the empirical fact of the unique outcomes of measurements, the world continuum theory needs an actual configuration, just like Bohmian mechanics, but it is taken as epistemic rather than ontic. It specifies not the actual configuration of the universe, but rather the configuration that is actual to a particular instance of an observer. With respect to his or her experience, and only so, there is a unique measurement outcome and an actual configuration of the universe. In essence, a complete objective description of Nature that complies with the empirical facts, requires also taking into account the subjective experience of Nature.

Acknowledgments There have been many enlightening and stimulating discussions with Rainer Plaga, Hrvoje Nikolic, Lev Vaidman, Delyan Zhelyasov, Jens Eisert, and Jan Michel. I also thank the reviewer for his or her thorough criticism that inspired considerable improvements. 


\section{References}

1. Albert, D., Loewer, B.: Interpreting the many worlds interpretation. Synthese 77(2), 195-213 (1988)

2. Barrett, J.: Everett's pure wave mechanics and the notion of worlds. Eur. J. Philos. Sci., 1-26 (2011). doi:10.1007/ s13194-011-0023-9

3. Bohm, D.: A suggested interpretation of the quantum theory in terms of "Hidden" variables. I. Phys. Rev. 85(2), 166-179 (1952a)

4. Bohm, D.: A suggested interpretation of the quantum theory in terms of "Hidden" variables. II. Phys. Rev. 85(2), 180-193 (1952b)

5. Boström, K.J.: Combining Bohm and Everett: axiomatics for a standalone quantum mechanics. arXiv:1208.5632 (2012)

6. Brown, H.: Comment on Valentini “De Broglie-Bohm pilot-wave theory: many worlds in denial?”. Arxiv preprint arXiv:0901.1278 (2009)

7. de Finetti, B.: The logic of probability. Philos. Stud. 77(1), (1995 [1936])

8. Deotto, E., Ghirardi, G.: Bohmian mechanics revisited. Found. Phys. 28, 1-30 (1998). doi:10.1023/A:1018752202576

9. Deutsch, D.: Comment on lockwood. Br. J. Philos. Sci. 47(2), 222-228 (1996)

10. Deutsch, D.: Quantum theory of probability and decisions. Proc. Math. Phys. Eng. Sci. 455(1988), 3129-3137 (1999)

11. Deutsch, D.: Vindication of quantum locality. Proc. R. Soc. A Math. Phys. Eng. Sci. 468(2138), 531-544 (2012)

12. DeWitt, B.S.: Quantum mechanics and reality. Phys. Today 23(9), 30-35 (1970)

13. Dowker, F., Kent, A.: On the consistent histories approach to quantum mechanics. J. Stat. Phys. 82, 1575-1646 (1996). doi:10. 1007/BF02183396

14. Dürr, D., Goldstein, S., Zanghí, N.: Quantum equilibrium and the origin of absolute uncertainty. J. Stat. Phys. 67, 843-907 (1992). doi:10.1007/BF01049004

15. Earman, J.: Determinism: what we have learned and what we still don't know. In: Campbell, J.K., O'Rourke, M., Shier, D. (eds.) Determinism, Freedom, and Agency. MIT Press, Cambridge (2004)

16. Elitzur, A., Vaidman, L.: Quantum mechanical interaction-free measurements. Found. Phys. 23, 987-997 (1993). doi:10.1007/ BF00736012

17. Everett, H.: "Relative State" formulation of quantum mechanics. Rev. Mod. Phys. 29(3), 454-462 (1957)

18. Everett, H.: The theory of the universal wavefunction. In: DeWitt, B., Graham, R. (eds.) The Many-Worlds Interpretation of Quantum Mechanics. Princeton University Press, Princeton (1973)

19. Farhi, E., Goldstone, J., Gutmann, S.: How probability arises in quantum mechanics. Ann. Phys. 192(2), 368-382 (1989)

20. Goldstein, S: Bohmian mechanics. The Stanford Encyclopedia of Philosophy (2009). http://plato.stanford.edu/archives/spr2009/ entries/qm-bohm/

21. Hall, M.J., Deckert, D.-A., Wiseman, H.M.: Quantum phenomena modeled by interactions between many classical worlds. Phys. Rev. X 4, 041013 (2014)

22. Hartle, J.B.: Quantum mechanics of individual systems. Am. J. Phys. 36(8), 704-712 (1968)

23. Holland, P.: Computing the wavefunction from trajectories: particle and wave pictures in quantum mechanics and their relation. Ann. Phys. 315(2), 505-531 (2005)

24. Kent, A.: Against many-worlds interpretations. Int. J. Mod. Phys. A 05(09), 1745-1762 (1990)

25. Kripke, S.: Naming and Necessity. Wiley-Blackwell, Oxford (1981)

26. de Laplace, P.S.: Théorie analytique des probabilités. Courcier, Paris (1814)

27. de Laplace, P.S.: Philosophical Essay On Probabilities. Wiley, New York (1902)

28. Lockwood, M.: 'Many minds'. interpretations of quantum mechanics. Br. J. Philos. Sci. 47(2), 159-188 (1996)

29. Madelung, E.: Quantentheorie in hydrodynamischer form. Zeitschrift für Physik 40(3-4), 322-326 (1927)

30. McInerney, P.K.: The nature of a person-stage. Am. Philos. Q. 28(3), 227-235 (1991)

31. Monton, B.: Wave function ontology. Synthese 130(2), 265-277 (2002)

32. Nikolić, H.: Quantum mechanics: myths and facts. Found. Phys. 37, 1563-1611 (2007)

33. Omnès, R.: Consistent interpretations of quantum mechanics. Rev. Mod. Phys. 64, 339-382 (1992)

34. Oriols, X., Mompart, J.: Overview of Bohmian mechanics. In: Oriols, X., Mompart, J. (eds.) Applied Bohmian Mechanics: From Nanoscale Systems to Cosmology (Chapter 1), pp. 15-147. Pan Stanford Publishing Pte. Ltd, Singapore (2012)

35. Poirier, B.: Bohmian mechanics without pilot waves. Chem. Phys. 370(1-3), 4-14 (2010)

36. Rae, A.I.: Everett and the born rule. Stud. Hist. Philos. Sci. Part B Stud. Hist. Philos. Mod. Phys. 40(3), $243-250$ (2009)

37. Saunders, S.: Time, quantum mechanics, and probability. Synthese 114(3), 373-404 (1998)

38. Saunders, S., Wallace, D.: Branching and uncertainty. Br. J. Philos. Sci. 59(3), 293-305 (2008)

39. Schiff, J., Poirier, B.: Communication: quantum mechanics without wavefunctions. J. Chem. Phys. 136(3), 031102 (2012)

40. Sebens, C.: Quantum mechanics as classical physics. arXiv:1403.0014 (2014)

41. Squires, E.J.: On an alleged "proof" of the quantum probability law. Phys. Lett. A 145(2-3), 67-68 (1990)

42. Tegmark, M.: The interpretation of quantum mechanics: many worlds or many words? Fortschritte der Physik 46(6-8), 855-862 (1998)

43. Tipler, F.J.: What about quantum theory? Bayes and the Born interpretation (2006)

44. Vaidman, L.: On the realization of interaction-free measurements. Quantum Opt. J. Eur. Opt. Soc. Part B 6(3), 119 (1994)

45. Vaidman, L.: On schizophrenic experiences of the neutron or why we should believe in the many-worlds interpretation of quantum theory. Int. Stud. Philos. Sci. 12(3), 245-261 (1998) 
46. Vaidman, L.: Many-worlds interpretation of quantum mechanics. The Stanford Encyclopedia of Philosophy (2008). http://plato. stanford.edu/archives/fall2008/entries/qm-manyworlds

47. Vaidman, L.: Counterfactuals in quantum mechanics. In: Greenberger, D., Hentschel, K., Weinert, F. (eds.) Compendium of Quantum Physics, pp. 132-136. Springer, Berlin (2009)

48. Vaidman, L.: Quantum theory and determinism. Quantum Stud. Math. Found. 1(1-2), 5-38 (2014)

49. Valentini, A.: Signal-locality, uncertainty, and the subquantum H-theorem. I. Phys. Lett. A 156(1-2), 5-11 (1991a)

50. Valentini, A.: Signal-locality, uncertainty, and the subquantum H-theorem. II. Phys. Lett. A 158(1-2), 1-8 (1991b)

51. Valentini, A.: De Broglie-Bohm pilot-wave theory: many worlds in denial? Arxiv preprint arXiv:0811.0810 (2008)

52. Varzi, A.: Mereology. In: Zalta, E.N. (ed.) The Stanford Encyclopedia of Philosophy. Stanford University (2014). http://plato. stanford.edu/archives/spr2014/entries/mereology

53. von Neumann, J.: Mathematische Grundlagen der Quantenmechanik. Springer, Berlin (1932)

54. Wallace, D.: The quantum measurement problem: state of play. In: Rickles, D. (ed.) The Ashgate Companion to the New Philosophy of Physics (chapter 1). Ashgate (2008)

55. Wallace, D.: Decoherence and ontology. In: Saunders, S., J, Barrett, Kent, A., Wallace, D. (eds.) Many Worlds? Everett, Quantum Theory, and Reality, pp. 53-72. Oxford University Press, Oxford (2010)

56. Werner, F.: Transcript of conference on the foundations of quantum mechanics, Xavier University Cincinnati, 1-October-1962, with Everett's remarks. http://hdl.handle.net/10575/1299 (1962) 